International Journal of Applied and Computational Mathematics (Springer, Germany)

https://www.springer.com/journal/40819

Accepted February $15^{\text {th }} 2020$

\title{
Computation of non-isothermal thermo-convective micropolar fluid dynamics in a Hall MHD generator system with non-linear distending wall
}

\author{
O. Anwar Bég' ${ }^{1}$ M. Ferdows², M. Enamul Karim³ ${ }^{3}$ M. Maruf Hasan³ ${ }^{3}$ T.A. Bég ${ }^{4}$, \\ MD. Shamshuddin ${ }^{* *}$ and A. Kadir ${ }^{1}$ \\ ${ }^{1}$ Professor, Multi-Physical Engineering Sciences, Mechanical Engineering Department, School of \\ Science, Engineering and Environment (SEE), Salford University, M54WT, UK. \\ ${ }^{2}$ College of Engineering and Science, Louisiana Tech University, Ruston, 71270, USA. \\ ${ }^{3}$ Department of Applied Mathematics, Comilla University, Comilla-3503, Bangladesh. \\ ${ }^{4}$ Computational Mechanics and Renewable Energy Research, Dickenson Rd., Manchester, M13, UK. \\ $5^{*}$ Department of Mathematics, Vaagdevi College of Engineering, Warangal, Telangana, India. \\ *Corresponding author: shamshuddin_md@vaagdevi.edu.in
}

\begin{abstract}
A theoretical model for steady non-isothermal convective heat transfer in non-Newtonian magnetized micropolar gas flow from a non-linear stretching/contracting wall in the presence of strong magnetic field is presented, as a simulation of an MHD (magnetohydrodynamic) Hall energy generator. Subsonic flow is considered, and compressibility effects neglected. The strength of the magnetic field which is applied in the general case obliquely to the wall is sufficient to invoke the collective effects of Hall current and Ohmic heating (Joule dissipation). Viscous heating is also included in the energy balance. Deploying similarity transformations, the governing equations are normalized into nonlinear ordinary differential equations with associated boundary conditions. The non-linear boundary value problem thus posed is then solved computationally with Nachtsheim-Swigert iteration technique along with the fourth-fifth order Runge-Kutta integration method (RKM). Verification of solutions is obtained with the semi-analytical Homotopy analysis method (HAM). Further validation is conducted with the semi-numerical Adomian Decomposition Method (ADM). In both cases excellent agreement is obtained with the Runge-Kutta shooting quadrature solutions. Additional validation is conducted with earlier Newtonian studies in the absence of micropolar, Hall current and dissipation effects. The influence of local Grashof number, local Hartmann number, Eringen microrotational parameter, Eringen coupling vortex parameter, Prandtl number and Eckert number on non-dimensional velocity components (primary, secondary and angular) and temperature within the boundary layer are graphically illustrated and interpreted at length. Furthermore, the effects of the thermophysical (e.g. non-isothermal power law index), electromagnetic parameters (e.g. Hall parameter) and geometric parameter (wall extension/contraction parameter) on the skinfriction coefficient (i.e. primary and secondary shear stress and wall couple stress) and surface heat transfer rate (Nusselt number) are evaluated. The study is relevant to near wall transport phenomena in novel MHD Hall power generators.
\end{abstract}

Keywords: Hall MHD power generators, Non-isothermal; Ohmic dissipation; Eringen micropolar gas dynamics; numerical solutions.

\section{Introduction}

MHD (magnetohydrodynamics) has garnered significant attention in renewable energy systems for a plethora of applications including space travel [1], ocean resources [2,3], coalfired power stations [4] and materials processing [5]. In novel designs for Mars missions [6] engineers have established that the ionization sheath encapsulating the spacecraft can be 
diminished markedly via MHD power generation, since the strength (and therefore damaging impact) of the ionization sheath is largely suppressed in key zones as electrons and ions are re-directed by the magnetic field and harvested at the electrodes. The redirected energy can be channelled into powering the vehicle. Since atmospheres generally contain debris and suspensions (cosmic dust), they exhibit certain non-Newtonian effects. The fluids extracted and employed in MHD re-direction systems therefore cannot be analysed as merely Newtonian (Navier-Stokes) viscous fluids. Although numerous nonNewtonian models exist for simulating complex shear stress-strain characteristics of working fluids in these proposed MHD devices, including Oldroyd-B [7], Maxwell [8], Jefferys [9] and power-law models, they all neglect micro-structural features of the fluids i.e. suspended particle spin. They are also generally restricted to liquids and not applicable to gaseous media. The spin effect can exert a profound influence on generator efficiency and heat transfer rates. In the 1960s a new branch of fluid dynamics was pioneered by the late A.C. Eringen, an engineering professor at Princeton University, USA. Micro-morphic fluid dynamics or "micro-fluids" [10] were introduced to mimic the gyratory motions of suspended particles. The initial theory was extremely complex, involving six times as many balance equations as in the classical Navier-Stokes theory. Eringen [11] therefore developed a specialized version of microfluids termed micropolar fluids. These fluids could simulate non-deformable micro-constituents and exhibited a constitutive equation with a nonsymmetrical stress tensor. These fluids respond to micro-rotational motions and spin inertia, and therefore can support couple stress and distributed body torque which are not achievable with the classical Navier-Stokes equations or the viscoelastic flow models. Micropolar fluid theory was subsequently applied to many branches of rheology including physiological fluids (blood containing corpuscles), colloidal suspensions, paints, liquid crystal suspensions, concentrated silica particle suspensions, oils containing very fine suspensions, industrial contaminants containing toxic chemicals, lubricants, organic/inorganic hybrid nano-composites and coastal sediments. The present study aims to further extend the applications to magnetic energy generators, an area which has not received significant attention from the viewpoint of micropolar gas dynamics. A great advantage of micropolar theory (which has been extensively validated experimentally see e.g. [12]) is that it can retrieve Newtonian model solutions as a very special elementary case. It also lends itself extremely well to boundary layer phenomena and is therefore ideal for simulating near wall flows in MHD ducts. A comprehensive review of over nearly five decades of theoretical and computational studies of micropolar flow, heat and mass transfer has been provided in the monograph by Bég et al. [13], the first work to do so. Some important studies of micropolar propulsion flows include Guram and Smith [14] who studied stagnation point flow of a micropolar fluid with strong and weak interactions. Gorla and Takhar [15] investigated the boundary layer flow of a micropolar fluid on a rotating axisymmetric surface with a concentrated heat source. Char and Chang [16] analyzed the effect of wall conduction on laminar natural convection heat transfer of micropolar fluids along a vertical flat plate. Takhar et al. [17] employed a finite element method for the flow and heat transfer in the micropolar fluid between two porous disks. El-Arabawy [18] carried out an analysis to study the effect of suction and injection on the flow and heat transfer characteristics for a continuous moving plate in a micropolar fluid in the presence of radiation. Hassanien et al. [19] found that, compared with Newtonian fluids, micropolar fluids enhance the skin friction and reduce heat transfer rate. Bég et al. [20] investigated boundary layer flow of micropolar fluids with double-diffusive convection from a spherical body. Damesh et al. [21] presented a numerical investigation of the natural heat transfer problem of a micropolar boundary layer flow near a vertical surface with constant heat flux. Bég et al. [22] used a homotopy method to simulate the squeezing hydrodynamics of a magnetized micropolar lubricant in a shock 
absorber system. Zueco et al. [23] analyzed the boyancy-driven micropolar propulsion in a vertical tube. Rahman [24] analyzed electric conductivity effects on a micropolar fluid flow from an oblique permeable flat plate in a permeable medium. Sherief et al. [25] considered the unsteady motion of an incompressible micropolar fluid filling a half-space bounded by a horizontal suddenly mobilized infinite plate. Numerous magnetohydrodynamic transport problems of micropolar fluids have also been reviewed in Bég [26] including subsonic and supersonic micropolar magneto-gas dynamic flows.

In the above-mentioned studies, the effect of Hall current has not been taken into consideration. Elie et al. [27] stressed the importance of incorporating Hall effects in thermosyphonic (and other) MHD propulsion systems e.g. those exploiting electrolyte working solutions, and observed that the Hall parameter, which is a property of the fluid of the electrolyte liquid, may attain very high values if not controlled and manifest in a significant loss in power output at the electrodes due to electron drift in the fluid leading to generation of current in an axial direction at the expense of the current flowing in the transverse direction between the electrodes. Several investigations have therefore addressed Hall current effects in magnetohydrodynamic transport, and an extensive review is provided in Bég et al. [28]. Aurangzaib and Shafie [29] investigated the effects of Soret and Dufour on combined heat and mass transfer in magnetic mixed convection in porous media with heat source, chemical reaction, Hall current and also radiative flux effects. Ferdows et al. [30] presented a similarity solution for the heat transfer flow considering slip conditions along with Hall and ion-slip currents. Bég et al. [31] employed a network simulation electrothermal code to simulate rotating plasma flow with Hall current in a porous medium. Kumar and Chand [32] presented a theoretical analysis of an unsteady hydromagnetic free convection flow of viscoelastic fluid through porous media. Ghosh et al. [33] obtained analytical and computational solutions for Maxwell displacement current and Hall current effects on rotating oblique magneto-hydrodynamic flow, considering ionized hydrogen as the propellant. Raju [34] studied unsteady magnetohydrodynamics flow of an electrically conducting fluid between two horizontal parallel non-conducting plates, where the lower one is stretching sheet and the upper one is oscillating porous plate in the presence of a transverse magnetic field and the effects of Hall current. Bég et al. [35] used multiple finite difference algorithms (Keller box and Nakamura tridoagonal) to compute the twodimensional non-isothermal, magnetohydrodynamic free convection boundary layer flow and heat transfer in a non-linear permeable material with Hall currents, ion slip, viscous heating and Ohmic dissipation effects. Ghara et al. [36] analyzed the unsteady MHD Couette flow of a viscous fluid between two infinite non-conducting horizontal porous plates with the both Hall currents and ion-slip. Hayat and Hendi [37] reported homotopy solutions for double-diffusive reactive magnetized three-dimensional axisymmetric flow of a viscous fluid between radially stretching sheets with Hall and ion-slip currents and thermal and magnetic dissipation. Motsa and Shateyi [38] considered Hall and variable thermal diffusivity effects on magnetomicropolar reactive heat, and mass transfer with suction through a porous medium using successive linearization method (SLM) together with a Chebyshev collocation method.

Thusfar to the authors' knowledge, the magnetohydrodynamic transport of micropolar fluid from an extending wall with Hall current and viscous/Ohmic dissipation has not been considered in the engineering science literature. The present article studies this problem and employs a variety of numerical algorithms to solve the resulting non-dimensional multiple degree non-linear coupled boundary value problem. Careful appraisal of Hall and other effects on the three-dimensional flow is addressed. The study is relevant to novel MHD 
propulsion systems for space travel and may also find applications in naval and marine MHD energy conversion utilizing micropolar conducting gases.

\section{Mathematical formulation}

We consider steady, incompressible and electrically conducting, micropolar fluid (ionized plasma) flow and heat transfer in the near-wall zone of an MHD Hall generator. A schematic describing the fundamentals of MHD generators is shown in Fig. 1a. The wall considered is non-linearly stretching and orientated along the $x$-direction. The flow under consideration is also subjected to a strong transverse magnetic field $B_{0}$ of constant intensity along the $y$-axis, as illustrated in Fig $\mathbf{1 b}$.

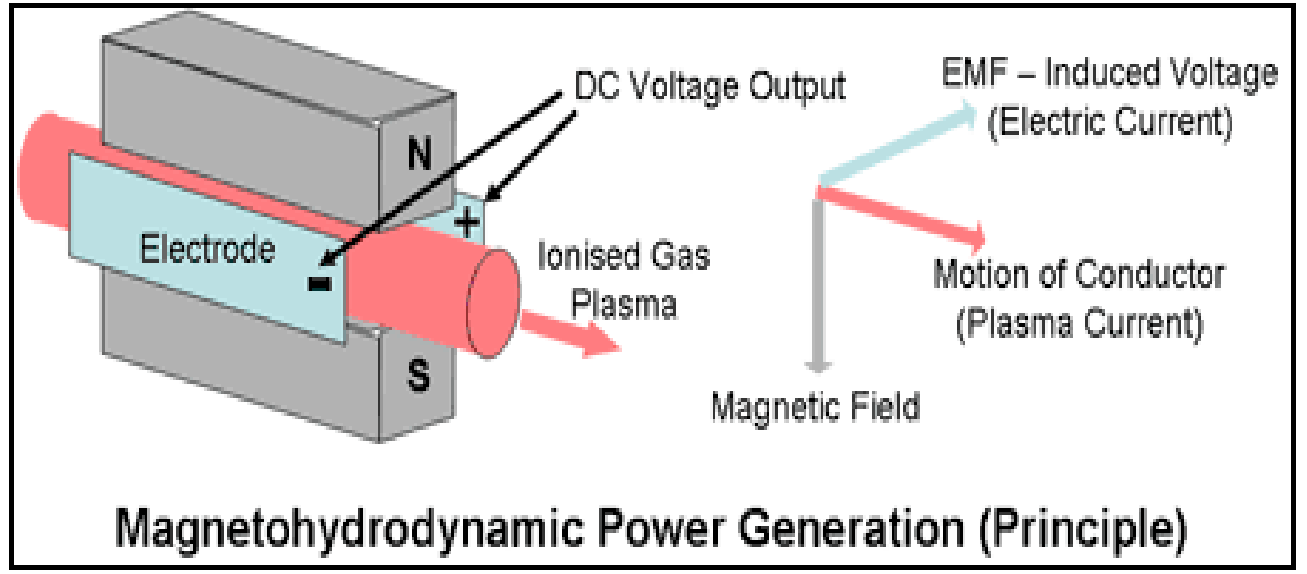

Fig 1a: MHD energy generation [Bég et al. [28]]

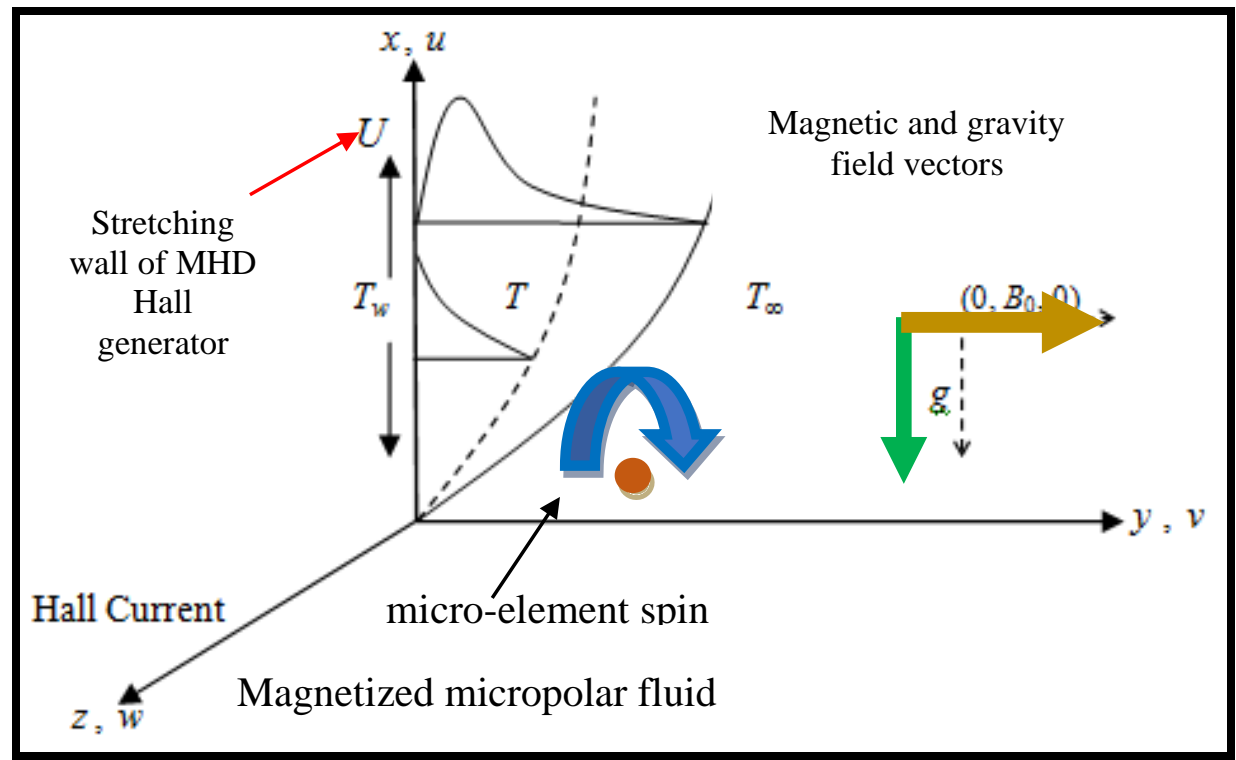

Fig 1b. Model for nonlinear MHD Hall generator micropolar flow

The velocity component $u$ on a stretching sheet is proportional to its distance from the leading edge of the boundary layer. Generally, Hall current influences an electrically conducting fluid in the presence of a magnetic field. The effect of Hall current gives rise to a force in the $z$-direction, which induces a crossflow in the $z$-direction and hence the flow 
becomes three-dimensional. We assume that all the fluid properties are isotropic and constant. The conservation equations for generalized transient flow of incompressible micropolar fluids are $[11,13]$ :

$\nabla \cdot \overline{\boldsymbol{V}}=0$

$\rho\left(\frac{\partial \overline{\boldsymbol{V}}}{\partial t}+\overline{\boldsymbol{V}} \cdot \nabla \overline{\boldsymbol{V}}\right)=-\nabla p+m_{1} \nabla \times \overline{\boldsymbol{V}}+\left(\mu+m_{1}\right) \nabla^{2} \overline{\boldsymbol{V}}$,

$\rho j\left(\frac{\partial \overline{\boldsymbol{v}}}{\partial t}+\overline{\boldsymbol{V}} \cdot \nabla \overline{\boldsymbol{v}}\right)=-2 m_{1} \overline{\boldsymbol{v}}+m_{1} \nabla \times \overline{\boldsymbol{V}}-\gamma(\nabla \times \nabla \times \overline{\boldsymbol{v}})+(\eta+\chi+\gamma) \nabla(\nabla \cdot \overline{\boldsymbol{v}})$,

where $\overline{\boldsymbol{V}}$ is the velocity vector, $\overline{\mathbf{v}}$ is the micro-rotation (micro-element angular i.e. spin velocity) vector, $\rho$ is the fluid density, $j$ is the micro-gyration parameter, $p$ is the fluid pressure, $\gamma, \chi, \eta$ are the coefficients of the viscosities and $m_{1}, \mu$ are coefficients of the vortex and shear viscosity, respectively. The parameters, $\mu, m_{1}, \gamma, \chi$ and $\eta$ are subject to the following thermodynamic constraints:

$2 \mu+m_{1} \geq 0, m_{1} \geq 0,3 \eta+\gamma+\chi \geq 0, \quad \gamma \geq|\chi|$.

Under the usual boundary layer and Boussinesq approximations, the governing equations in an $(x, y, z)$ coordinate system for the MHD flow under consideration can be extracted and modified accordingly. Here Eqns. (5-8) are derived by reducing the full vector equations (13 ) and only retaining the relevant terms for the current problem based on boundary layer and Boussinesq approximations (as already stated) and adding any relevant supplementary terms e.g. viscous heating and Hall currents. Only the spin (micro-rotation) in the $x-y$ plane is retained and therefore only a single angular momentum (micro-rotation) equation is required (and not the three that feature in the general micropolar model [11]). The emerging equations effectively amalgamate the models given in [24] and [30] as stated below:

$$
\begin{aligned}
& \frac{\partial u}{\partial x}+\frac{\partial v}{\partial y}=0 \\
& u \frac{\partial u}{\partial x}+v \frac{\partial u}{\partial y}=v \frac{\partial^{2} u}{\partial y^{2}}+g \beta\left(T-T_{\infty}\right)-\frac{B_{0}}{\rho} J_{z}+K_{1} \frac{\partial N}{\partial y} \\
& u \frac{\partial w}{\partial x}+v \frac{\partial w}{\partial y}=v \frac{\partial^{2} w}{\partial y^{2}}+\frac{B_{0}}{\rho} J_{x} \\
& \frac{G_{1}}{K} \frac{\partial^{2} N}{\partial y^{2}}-2 N-\frac{\partial u}{\partial y}=0 \\
& u \frac{\partial T}{\partial x}+v \frac{\partial T}{\partial y}=\frac{\kappa}{\rho C_{p}} \frac{\partial^{2} T}{\partial y^{2}}+\frac{\sigma \mu_{e} B_{0}{ }^{2} \lambda}{\rho C_{p}\left(1+m^{2} \lambda^{2}\right)}\left(u^{2}+w^{2}\right)
\end{aligned}
$$

The initial and boundary conditions are

$$
\left.\begin{array}{ll}
u=U=B x^{n}, v=0, w=0, \quad N=0, T=T_{\mathrm{w}}=T_{\infty}+A x^{\gamma} & \text { at } y=0 \\
u \rightarrow 0, \quad w \rightarrow 0, \quad N \rightarrow 0, \quad T \rightarrow T_{\infty} & \text { as } y \rightarrow \infty
\end{array}\right\}
$$

Here positive or negative $n$ indicates acceleration or deceleration of the wall from the entry point (origin) whereas $n=0$ is the case of a stationary wall, respectively, $(u, v, w)$ are the fluid velocity components in the $x, y, z$-directions respectively, $N$ is the micro-element 
micro-rotation, $T$ is the temperature, $J_{x}=\frac{\sigma \mu_{e} B_{0} \lambda}{1+m^{2} \lambda^{2}}(m \lambda u-w)$ and $J_{z}=\frac{\sigma \mu_{e} B_{0} \lambda}{1+m^{2} \lambda^{2}}(u+m \lambda w)$ are the currents along the $x$-and $z$-axis respectively, $\sigma$ is the electric conductivity of micropolar fluid, $\mu_{e}$ is the viscosity of fluid, $B_{0}$ is the uniform magnetic field strength, $m=\omega_{e} \tau_{e}$ is the Hall parameter and $\lambda=\cos \alpha$ where $\alpha$ is the angle between the direction of the strong uniform magnetic field $\vec{B}$ and the plane transverse to the plate (the latter is assumed to be electrically non-conducting), $v$ is the kinematic viscosity, $\beta$ is the volumetric coefficient of thermal expansion, $\rho$ is the fluid density, $K$ is the Eringen vortex viscosity, $G_{1}$ is the Eringen spin gradient viscosity, $\kappa$ is the thermal conductivity of the fluid, $C_{p}$ denotes the specific heat at constant pressure and $A$ and $\gamma$ are constants. Numerical solutions of the primitive boundary value problem defined by Eqns. (5)-(10) generally necessitate computationally rigorous codes e.g. finite difference or finite element. They also yield only very general information regarding the flow fields and preclude the opportunity to invoke dimensionless parameters which can provide detailed understanding of the thermophysical and magnetic field effects. It is therefore pertinent to transform the coordinate system to a non-dimensional one and this is achieved readily via non-similar transformations, simultaneously eliminating one of the independent variables and reducing the partial differential equation system into an ordinary differential one. Introducing the following transformation variables.

$$
\begin{aligned}
& \eta=y\left[\frac{B(n+1)}{2 v}\right]^{\frac{1}{2}} x^{\frac{n-1}{2}}, u=B x^{n} f^{\prime}(\eta), v=-\left[B v\left(\frac{n+1}{2}\right)\right]^{\frac{1}{2}} x^{\frac{n-1}{2}}\left[f+\frac{n-1}{n+1} \eta f^{\prime}\right], \\
& w=B x^{n} g(\eta), \quad N=B\left[\frac{B(n+1)}{2 v}\right]^{\frac{1}{2}} x^{\frac{3 n-1}{2}} h(\eta), \quad \theta(\eta)=\frac{T-T_{\infty}}{T_{w}-T_{\infty}}
\end{aligned}
$$

Therefore. the transformed equations using the above parameters are converted to a ninth order ordinary differential boundary value problem defined by:

$$
\begin{aligned}
& f^{\prime \prime \prime}+f f^{\prime \prime}-N_{1} h^{\prime}-\frac{2}{n+1}\left[n f^{\prime 2}-G r \theta+\frac{M \lambda}{1+m^{2} \lambda^{2}}\left(f^{\prime}+m \lambda g\right)\right]=0 \\
& g^{\prime \prime}+f g^{\prime}-\frac{2}{n+1}\left[n f^{\prime} g-\frac{M \lambda}{1+m^{2} \lambda^{2}}\left(m \lambda f^{\prime}-g\right)\right]=0 \\
& G\left(\frac{n+1}{2}\right) h^{\prime \prime}-2 h-f^{\prime \prime}=0 \\
& \frac{1}{\operatorname{Pr}} \theta^{\prime \prime}+f \theta^{\prime}-\frac{2}{n+1}\left[\gamma f^{\prime} \theta-\frac{M \lambda}{1+m^{2} \lambda^{2}} \cdot E c\left(f^{\prime 2}+g^{2}\right)\right]=0
\end{aligned}
$$

The corresponding initial and boundary conditions contract to:

$$
\left.\begin{array}{l}
f=0, f^{\prime}=1, \quad g=0, h=0, \theta=1 \quad \text { at } \eta=0 \\
f^{\prime} \rightarrow 0, \quad g \rightarrow 0, \quad h \rightarrow 0, \quad \theta \rightarrow 0 \quad \text { as } \eta \rightarrow \infty
\end{array}\right\}
$$

Here, $G r=g \beta\left(T_{w}-T_{\infty}\right) x / U^{2}$ is the local Grashof number, $M=\sigma \mu_{e} B_{0}{ }^{2} x / \rho U$ is the local magnetic body force parameter (Hartmann number) in which $B_{0}=B / \sqrt{x}$ is the scaled magnetic field strength, $G=G_{1} B x^{n-1} / K v$ is the micro-rotational parameter, $N_{1}=K_{1} / v$ is the material (Eringen coupling) parameter, $\operatorname{Pr}=\rho v C_{p} / \kappa$ is the Prandtl number and $E c=U^{2} / C_{p}\left(T_{w}-T_{\infty}\right)$ is the Eckert number. The Grashof, magnetic and micro-rotational parameters are local similarity 
parameters which are required in the non-dimensionalization of the model. This is a perfectly valid approach which has been used by many scientists in heat transfer and magnetofluid dynamics. It retains the requisite validity of the physics and solutions presented in our paper are locally autonomous (i. e. independent of information from other streamwise locations) as noted by Sparrow and $\mathrm{Yu}[39]$.

The parameters of engineering interest for MHD energy generators include the wall skinfriction components for the primary and secondary velocities, and the local Nusselt number $(\mathrm{Nu})$. The wall skin-friction components $\tau_{w x}$ and $\tau_{w z}$ for the primary and secondary velocities respectively are given by:

$$
\begin{aligned}
& \tau_{w x}=\left.\mu \frac{\partial u}{\partial y}\right|_{y=0}=\mu U x^{-\frac{1}{2}\left(\frac{U(n+1)}{2 v}\right)^{\frac{1}{2}} f^{\prime \prime}(0)} \\
& \tau_{w z}=\left.\mu \frac{\partial w}{\partial y}\right|_{y=0}=\mu U x^{-\frac{1}{2}\left(\frac{U(n+1)}{2 v}\right)^{\frac{1}{2}} g^{\prime}(0)}
\end{aligned}
$$

Hence the skin-friction coefficients $C_{f x}$ and $C_{f z}$ for the primary and secondary velocities respectively, i.e. primary and secondary shear stress, are given by:

$$
\begin{aligned}
& C_{f x}=\frac{\tau_{w x}}{\frac{1}{2} \rho U^{2}}=\sqrt{2(n+1)} \operatorname{Re}^{-\frac{1}{2}} f^{\prime \prime}(0) \\
& C_{f z}=\frac{\tau_{w z}}{\frac{1}{2} \rho U^{2}}=\sqrt{2(n+1)} \operatorname{Re}^{-\frac{1}{2}} g^{\prime}(0)
\end{aligned}
$$

Now the local Nusselt number is given by

$$
N u_{x}=-\left.\frac{x}{\left(T_{w}-T_{\infty}\right)} \frac{\partial T}{\partial y}\right|_{y=0}=-\left(\frac{\operatorname{Re}(n+1)}{2}\right)^{\frac{1}{2}} \theta^{\prime}(0)
$$

Thus, from the above definition we have $C_{f x} \propto f^{\prime \prime}(0), C_{f z} \propto g^{\prime}(0)$ and $N u_{x} \propto-\theta^{\prime}(0)$.

\section{Numerical computation with shooting scheme}

The numerical solutions of the non-linear differential equations (12) to (15) under the boundary conditions (16) have been performed by applying a hybrid shooting quadrature method namely the Nachtsheim and Swigert [40] iteration technique (guessing the missing values) along with a fourth-fifth order Runge-Kutta-Fehlberg iteration scheme. We have chosen a step size $\Delta \eta=0.01$ to satisfy the convergence criterion of $10^{-5} \mathrm{in}$ all cases. The value of $\eta_{\infty}$ has been found to each iteration loop by $\eta_{\infty}=\eta_{\infty}+\Delta \eta$. MAPLE 17 symbolic software is employed to execute the computations via the "dsolve" library [41]. This approach has been extensively implemented in recent years and speedily solves very strongly nonlinear problems including magnetized nanofluid flow [42] and bioconvection slip flows [43]. A Runge-Kutta-Fehlberg algorithm is employed which utilizes a collocation method in which a finite-dimensional space of candidate solutions is selected (usually, polynomials up to a certain degree) and a number of points in the domain (called collocation points), and a 
solution selected which satisfies the given equation at the collocation points. The algorithm is adaptive since it adjusts the quantity and location of grid points during iteration and thereby constrains the local error within acceptable specified bounds. In the current problem, the asymptotic boundary conditions given in Eqn. (16) are replaced by a finite value of 8 . The choice of infinity must be selected judiciously to ensure that all numerical solutions approach the asymptotic values correctly. The selection of a sufficiently large value for infinity is absolutely critical in sustaining the desired accuracy in boundary layer flows and is a common pitfall encountered in numerous studies. The stepping formulae used to solve Eqns. (12)-(15) under conditions (16) with this computational shooting algorithm are given below [44]:

$$
\begin{aligned}
& k_{0}=f\left(x_{i}, y_{i}\right) \\
& k_{1}=f\left(x_{i}+\frac{h}{4}, y_{i}+\frac{h}{4} k_{0}\right) \\
& k_{2}=f\left(x_{i}+\frac{3 h}{8}, y_{i}+\left(\frac{3}{32} k_{0}+\frac{9}{32} k_{1}\right) h\right) \\
& k_{3}=f\left(x_{i}+\frac{12 h}{13}, y_{i}+\left(\frac{1932}{2197} k_{0}-\frac{7200}{2197} k_{1}+\frac{7296}{2197} k_{2}\right) h\right) \\
& k_{4}=f\left(x_{i}+h, y_{i}+\left(\frac{439}{216} k_{0}-8 k_{1}+\frac{3860}{513} k_{2}-\frac{845}{41047} k_{3}\right) h\right) \\
& k_{5}=f\left(x_{i}+\frac{h}{2}, y_{i}+\left(-\frac{8}{27} k_{0}+2 k_{1}-\frac{3544}{2565} k_{2}+\frac{1859}{4104} k_{3}-\frac{11}{40} k_{4}\right) h\right) \\
& y_{i+1}=y_{i}+\left(\frac{25}{216} k_{0}+\frac{1408}{2565} k_{2}+\frac{2197}{4104} k_{3}-\frac{1}{5} k_{4}\right) h \\
& z_{i+1}=z_{i}+\left(\frac{16}{135} k_{0}+\frac{6656}{12825} k_{2}+\frac{28561}{56430} k_{3}-\frac{9}{50} k_{4}+\frac{2}{55} k_{5}\right) h
\end{aligned}
$$

Here $y$ denotes fourth order Runge-Kutta phase and $z$ is the fifth order Runge-Kutta phase. An estimate of the error is achieved by subtracting the two values obtained. If the error exceeds a specified threshold, the results can be recalculated using a smaller step size. The approach to estimating the new step size is shown below:

$h_{\text {new }}=h_{\text {old }}\left(\frac{\varepsilon h_{\text {old }}}{2\left|z_{i+1}-y_{i+1}\right|_{5}}\right)^{1 / 4}$

\section{Validation with homotopy analysis method}

Since no practical benchmark exist in the literature for the present general model, we seek verification of solutions via two alternate techniques. The first of these, namely the Homotopy analysis method (HAM) has rapidly grown into a powerful alternative to traditional finite difference or finite element methods and is equally applicable to nonlinear systems of partial or ordinary differential equations. Liao [45] employed developed HAM via homotopy in topology to generate a general analytical-numerical method for nonlinear 
problems. The validity of HAM is independent of whether or not there exist small parameters in the considered equation(s). Therefore HAM can overcome the foregoing restrictions of perturbation methods. In recent years, HAM has been successfully employed to solve many types of non-linear problems in engineering sciences including rotating nanofluid dynamics [46], acoustical vibration [47] and swirling aerodynamics [48]. Denoting $f=F, g=G, h=H$ and $\theta=N$, in HAM we write the initial guesses and linear operators as:

$$
\begin{aligned}
& F_{0}(\eta)=\eta-1+e^{-\eta}, \quad G_{0}(\eta)=e^{-\eta}, \quad H_{0}(\eta)=e^{-\eta}, \quad N_{0}(\eta)=e^{-\eta}, \\
& L_{F}=\frac{d^{3} F}{d \eta^{3}}-\frac{d F}{d \eta}, L_{G}=\frac{d^{2} G}{d \eta^{2}}-G, L_{H}=\frac{d^{2} H}{d \eta^{2}}-H, L_{N}=\frac{d^{2} N}{d \eta^{2}}-N,
\end{aligned}
$$

with the following properties:

$$
\begin{array}{ll}
L_{F}\left(C_{1}+C_{2} e^{\eta}+C_{3} e^{-\eta}\right)=0, & L_{G}\left(C_{4} e^{\eta}+C_{5} e^{-\eta}\right)=0, \\
L_{G}\left(C_{6} e^{\eta}+C_{7} e^{-\eta}\right)=0, & L_{N}\left(C_{8} e^{\eta}+C_{9} e^{-\eta}\right)=0,
\end{array}
$$

here $C_{i}(i=1-9)$ are arbitrary constants. Let $q \in[0,1]$ represent an embedding parameter and $\hbar_{F}, \hbar_{G}, \hbar_{H}, \hbar_{N}$ denote the nonzero auxiliary linear operators and construct the following zeroth order deformation equations:

$$
\begin{aligned}
& (1-q) L_{f}\left[\hat{F}(\eta ; q)-F_{0}(\eta)\right]=q \hbar_{F} N_{F}^{*}[\hat{F}(\eta ; q)] \\
& (1-q) L_{G}\left[\hat{G}(\eta ; q)-G_{0}(\eta)\right]=q \hbar_{G} N_{G}^{*}[\hat{G}(\eta ; q), \hat{F}(\eta ; q)] \\
& (1-q) L_{H}\left[\hat{H}(\eta ; q)-H_{0}(\eta)\right]=q \hbar_{H} N_{H}^{*}[\hat{H}(\eta ; q), \hat{F}(\eta ; q)], \\
& (1-q) L_{N}\left[\hat{N}(\eta ; q)-N_{0}(\eta)\right]=q \hbar_{N} N_{N}^{*}[\hat{N}(\eta ; q), \hat{F}(\eta ; q)],
\end{aligned}
$$

The transformed boundary conditions are non-linear operators may then be defined. Taylor expansions of the approximations are then conducted, and auxiliary parameters are properly selected to achieve fast convergence of these series. The resulting problems at the $m^{\text {th }}$ order deformation are then formulated with associated boundary conditions and eventually a general solution of the Eqns. (12)-(15) is achieved in which $F_{m}^{*}(\eta)$, $G_{m}^{*}(\eta), H_{m}^{*}(\eta)$ and $N_{m}^{*}(\eta)$ are the particular solutions and the constants are to be determined by the boundary conditions. HAM achieves an analytical solution of the problem in series form. An important consideration is convergence of the series solution given by HAM which depends strongly upon auxiliary parameters $\hbar_{F}, \hbar_{G}, \hbar_{H}$ and $\hbar_{N}$. These parameters provide a convenient mechanism for adjusting and controlling the convergence region and convergence rate of the series solution. Therefore, in order to select appropriate values for these auxiliary parameters, the so called $\hbar_{F}, \hbar_{G}, \hbar_{H}$ and $\hbar_{N}$ curves are computed at $20^{\text {th }}$ order approximations to guarantee exceptional accuracy and is therefore adopted in all HAM numerical computations. The comparison of solutions via shooting quadrature and HAM is provided in Tables 1-9.

\section{Validation with ADM and published results}

As further verification of the shooting solutions, the boundary value problem has again been solved with another code, ADMicro, employing ADM, a different semi-numerical technique which employs Adomian polynomials to achieve very accurate solutions which 
may be evaluated using symbolic packages such as MATLAB. ADM has been used successfully by the authors in recent years to simulate magnetized lubrication problems [49] and viscoelastic extrusion flows [50]. ADM [51] deploys an infinite series solution for the unknown functions i.e. $f, g, h$ and $\theta$, in Eqns (12)-(16) and utilizes recursive relations. The present ordinary differential nonlinear boundary value problem (BVP) is re-written using the standard operator, following Shamshuddin et al. [52]:

$$
L u+R u+N u=g
$$

where $u$ is the unknown function, $L$ is the highest order derivative (assumed to be easily invertible), $R$ is a linear differential operator of order less than $L, N$ designates the nonlinear terms, and $g$ is the source term. Applying the inverse operator $L^{-1}$ to both sides of eqn. (37) and using the given conditions we obtain:

$u=v-L^{-1}(R u)-L^{-1}(N)$

where $v$ represents the terms arising from integrating the source term $g$ and from the auxiliary conditions. ADM defines the solution $u$ by the series:

$$
u=\sum_{n=0}^{\infty} u_{n}
$$

The solution for the non-linear terms is:

$$
N=\sum_{n=0}^{\infty} A_{n}
$$

Here $A_{n}$ are the Adomian polynomials which are evaluated via the following relation [49]:

$$
A_{n}=\frac{1}{n !}\left\{\frac{d^{n}}{d \lambda^{n}}\left[N \sum_{i=0}^{\infty} \lambda^{i} u_{i}\right]\right\}_{\lambda=0}
$$

If the nonlinear term is expressed as a nonlinear function $f(u)$, the Adomian polynomials are arranged into the form:

$$
\begin{aligned}
& A=f\left(u_{0}\right) \\
& A_{1}=u_{1} f^{(1)}\left(u_{0}\right) \\
& A_{2}=u_{2} f^{(1)}\left(u_{0}\right)+\frac{1}{2 !} u_{1}^{2} f^{(2)}\left(u_{0}\right) \\
& A_{3}=u_{3} f^{(1)}\left(u_{0}\right)+u_{1} u_{2} f^{(2)}\left(u_{0}\right)+\frac{1}{3 !} u_{1}^{3} f^{(3)}\left(u_{0}\right)
\end{aligned}
$$

The components $u_{0}, u_{1}, u_{2} \ldots$ are then determined recursively by using the relation:

$$
\begin{aligned}
& u_{0}=v \\
& u_{k+1}=-L^{-1} R u_{k}-L^{-1} A_{k} \quad k \geq 0
\end{aligned}
$$

where $u_{0}$ is referred to as the zeroth component. An $n$-components truncated series solution is finally obtained as:

$$
S_{n}=\sum_{n=0}^{\infty} u_{i} \text {. }
$$

Computations in MATLAB on an Octane SGI desktop workstation (dual processor) take seconds to perform and an accuracy of $10^{-5}$ is achieved throughout. The numerical values proportional to the skin-friction coefficients and the Nusselt-number are given in Tables 1-9 for different values of the thermo-physical, magnetic and micropolar (rheological) parameters for all three methods employed (RKM, HAM and ADM). The correlation is excellent. The tables bear testimony to the excellent accuracy of RKM as compared with other techniques. Confidence in all three algorithms is therefore justifiably high and this data provides a robust benchmark for future studies. To further confirm the accuracy of all three numerical solvers 
employed, a supplementary verification has been conducted. The present model reduces exactly to that of Gorla and Sidwai [53] when $M=0$ (non-magnetic), $\mathrm{N}_{1}=0$ (micropolar effects negated), Ec $=0$ (viscous heating ignored), Grashof number $(G r)=1$, Hall parameter $(m)=0, n=0$ (linear stretching), $G=0$ (micro-rotation neglected), $\gamma=0$ (isothermal) for $\operatorname{Pr}=$ 0.7. Equations (13) and (14) are neglected to correlate the model with [53]. Excellent agreement is achieved between all three methods and the Nakamura finite difference numerical solutions of Gorla and Sidawi [53], as shown in Table 10. This confirms beyond doubt the accuracy of our computations.

Table 1: Comparison of RKM, HAM and ADM numerical solutions for various Hartmann numbers $(M)$ with $N_{l}=0.2, G r=5.0, G=2.0, \operatorname{Pr}=0.72, E c=0.3, m=1.0, n=1.0, \gamma=0.02$ and $\lambda=1.0$.

\begin{tabular}{|c|c|c|c|c|c|c|c|c|c|c|c|c|}
\hline$M$ & $f^{\prime \prime}(0)$ & $f^{\prime \prime}(0)$ & $f^{\prime \prime}(0)$ & $g^{\prime}(0)$ & $g^{\prime}(0)$ & $g^{\prime}(0)$ & $h^{\prime}(0)$ & $h^{\prime}(0)$ & $h^{\prime}(0)$ & $-\theta^{\prime}(0)$ & $-\theta^{\prime}(0)$ & $-\theta^{\prime}(0)$ \\
$(\mathbf{R K M})$ & $(\mathbf{H A M})$ & $(\mathbf{A D M})$ & $(\mathbf{R K M})$ & $(\mathbf{H A M})$ & $(\mathbf{A D M})$ & $(\mathbf{R K M})$ & $(\mathbf{H A M})$ & $(\mathbf{A D M})$ & $(\mathbf{R K M})$ & $(\mathbf{H A M})$ & $(\mathbf{A D M})$ \\
\hline-1 & 1.3897 & 1.3898 & 1.3896 & -0.5486 & -0.5485 & -0.5487 & 0.0419 & 0.0418 & 0.0420 & 0.8470 & 0.8471 & 0.8472 \\
\hline 0 & 1.2398 & 1.2399 & 1.2397 & 0.0000 & 0.0000 & 0.0000 & 0.0456 & 0.0455 & 0.0457 & 0.6979 & 0.6980 & 0.6978 \\
\hline 1 & 1.0178 & 1.0179 & 1.0177 & 0.3929 & 0.3927 & 0.3930 & 0.0630 & 0.0629 & 0.0631 & 0.5530 & 0.5531 & 0.5529 \\
\hline 3 & 0.5257 & 0.5256 & 0.5258 & 0.9014 & 0.9015 & 0.9016 & 0.1057 & 0.1056 & 0.1058 & 0.2968 & 0.2969 & 0.2967 \\
\hline 5 & 0.0512 & 0.0513 & 0.0511 & 1.2005 & 1.2004 & 1.2006 & 0.1457 & 0.1456 & 0.1458 & 0.0953 & 0.0954 & 0.0952 \\
\hline 7 & -0.3812 & -0.3813 & -0.3811 & 1.3905 & 1.3904 & 1.3906 & 0.1803 & 0.1802 & 0.1805 & 0.0548 & 0.0549 & 0.0547 \\
\hline
\end{tabular}

Table 2: Comparison of RKM, HAM and ADM numerical solutions for various Eringen coupling parameters $\left(N_{1}\right)$ with $M=3.0, G r=5.0, G=2.0, \operatorname{Pr}=0.72, E c=0.3, m=1.0, n=1.0, \gamma=0.02$ and $\lambda=1.0$.

\begin{tabular}{|c|c|c|c|c|c|c|c|c|c|c|c|c|}
\hline$N_{I}$ & $\begin{array}{c}f^{\prime \prime}(0) \\
(\mathbf{R K M})\end{array}$ & $\begin{array}{c}f^{\prime \prime}(0) \\
\mathbf{( H A M )}\end{array}$ & $\begin{array}{c}f^{\prime \prime}(0) \\
\mathbf{( A D M})\end{array}$ & $\begin{array}{c}g^{\prime}(0) \\
\mathbf{( R K M )}\end{array}$ & $\begin{array}{c}g^{\prime}(0) \\
\text { (HAM }\end{array}$ & $\begin{array}{c}g^{\prime}(0) \\
(\mathbf{A D M})\end{array}$ & $\begin{array}{c}h^{\prime}(0) \\
(\mathbf{R K M})\end{array}$ & $\begin{array}{c}h^{\prime}(0) \\
(\mathbf{H A M})\end{array}$ & $\begin{array}{c}h^{\prime}(0) \\
(\mathbf{A D M})\end{array}$ & $\begin{array}{c}-\theta^{\prime}(0) \\
\text { (RKM) }\end{array}$ & $\begin{array}{c}-\theta^{\prime}(0) \\
\text { (HAM) }\end{array}$ & $\begin{array}{c}-\theta^{\prime}(0) \\
\text { (ADM }\end{array}$ \\
\hline 0.2 & 0.5257 & 0.5256 & 0.5258 & 0.9014 & 0.9015 & 0.9015 & 0.1057 & 0.1056 & 0.1058 & 0.2968 & 0.2970 & 0.2969 \\
\hline 0.5 & 0.5419 & 0.5420 & 0.5218 & 0.9023 & 0.9022 & 0.9021 & 0.1052 & 0.1051 & 0.1053 & 0.2967 & 0.2968 & 0.2966 \\
\hline 1.0 & 0.5692 & 0.5693 & 0.5691 & 0.9037 & 0.9036 & 0.9035 & 0.1045 & 0.1044 & 0.1046 & 0.2966 & 0.2965 & 0.2965 \\
\hline 2.0 & 0.6260 & 0.6261 & 0.6259 & 0.9068 & 0.9067 & 0.9069 & 0.1029 & 0.1028 & 0.1030 & 0.2962 & 0.2961 & 0.2963 \\
\hline 4.0 & 0.7488 & 0.7489 & 0.7487 & 0.9126 & 0.9128 & 0.9127 & 0.0998 & 0.0997 & 0.0999 & 0.2949 & 0.2950 & 0.2948 \\
\hline 8.0 & 1.0493 & 1.0494 & 1.0492 & 0.9226 & 0.9227 & 0.9225 & 0.0942 & 0.0941 & 0.0943 & 0.2877 & 0.2876 & 0.2879 \\
\hline
\end{tabular}

Table 3: Comparison of RKM, HAM and ADM numerical solutions for various Grashof numbers $(G r)$ with $M=3.0, N_{l}=0.2, G=2.0, \operatorname{Pr}=0.72, E c=0.3, m=1.0, n=1.0, \gamma=0.02$ and $\lambda=1.0$.

\begin{tabular}{|c|c|c|c|c|c|c|c|c|c|c|c|c|}
\hline$G r$ & $f^{\prime \prime}(0)$ & $f^{\prime \prime}(0)$ & $f^{\prime \prime}(0)$ & $g^{\prime}(0)$ & $g^{\prime}(0)$ & $g^{\prime}(0)$ & $h^{\prime}(0)$ & $h^{\prime}(0)$ & $h^{\prime}(0)$ & $-\theta^{\prime}(0)$ & $-\theta^{\prime}(0)$ & $-\theta^{\prime}(0)$ \\
$(\mathbf{R K M})$ & $(\mathbf{H A M})$ & $(\mathbf{A D M})$ & $\mathbf{( R K M )}$ & $\mathbf{H A M})$ & $(\mathbf{A D M})$ & $(\mathbf{R K M})$ & $(\mathbf{H A M})$ & $(\mathbf{A D M})$ & $(\mathbf{R K M})$ & $(\mathbf{H A M})$ & $(\mathbf{A D M})$ \\
\hline 0.0 & -1.6452 & -1.6451 & -1.6453 & 0.4835 & 0.4834 & 0.4836 & 0.3188 & 0.3189 & 0.3187 & 0.2507 & 0.2508 & 0.2509 \\
\hline 1.0 & -1.1814 & -1.1813 & -1.1814 & 0.6025 & 0.6023 & 0.6026 & 0.2638 & 0.2639 & 0.2637 & 0.3153 & 0.3152 & 0.3151 \\
\hline 2.0 & -0.7424 & -0.7423 & -0.7425 & 0.6932 & 0.6931 & 0.6933 & 0.2188 & 0.2189 & 0.2189 & 0.3292 & 0.3291 & 0.3291 \\
\hline 3.0 & -0.3144 & -0.3143 & -0.3146 & 0.7705 & 0.7704 & 0.7705 & 0.1784 & 0.1782 & 0.1783 & 0.3264 & 0.3262 & 0.3263 \\
\hline 5.0 & 0.5257 & 0.5256 & 0.5257 & 0.9014 & 0.9013 & 0.9015 & 0.1057 & 0.1059 & 0.1056 & 0.2968 & 0.2967 & 0.2969 \\
\hline 8.0 & 1.7744 & 1.7742 & 1.7745 & 1.0626 & 1.0625 & 1.0627 & 0.0081 & 0.0080 & 0.0082 & 0.2181 & 0.2180 & 0.2180 \\
\hline 10 & 2.6102 & 2.6103 & 2.6101 & 1.1555 & 1.1554 & 1.1556 & -0.0521 & -0.0520 & -0.0522 & 0.1505 & 0.1504 & 0.1503 \\
\hline
\end{tabular}


Table 4: Comparison of RKM, HAM and ADM numerical solutions for various micro-rotation parameters $(G)$ with $M=3.0, N_{1}=0.2, G r=5.0, \operatorname{Pr}=0.72, E c=0.3, m=1.0, n=1.0, \gamma=0.02$ and $\lambda=1.0$.

\begin{tabular}{|c|c|c|c|c|c|c|c|c|c|c|c|c|}
\hline$G$ & $\begin{array}{c}f^{\prime \prime}(0) \\
(\mathbf{R K M}\end{array}$ & $\begin{array}{c}f^{\prime \prime}(0) \\
(\mathbf{H A M}\end{array}$ & $\begin{array}{c}f^{\prime \prime}(0) \\
(\mathbf{A D M}\end{array}$ & $\begin{array}{c}g^{\prime}(0) \\
\mathbf{( R K M})\end{array}$ & $\begin{array}{c}g^{\prime}(0) \\
\mathbf{( H A M})\end{array}$ & $\begin{array}{c}g^{\prime}(0) \\
\mathbf{( A D M})\end{array}$ & $\begin{array}{c}h^{\prime}(0) \\
(\mathbf{R K M})\end{array}$ & $\begin{array}{c}h^{\prime}(0) \\
(\mathbf{H A M})\end{array}$ & $\begin{array}{c}h^{\prime}(0) \\
(\mathbf{A D M})\end{array}$ & $\begin{array}{c}-\theta^{\prime}(0) \\
(\mathbf{R K M})\end{array}$ & $\begin{array}{c}-\theta^{\prime}(0) \\
(\mathbf{H A M})\end{array}$ & $\begin{array}{c}-\theta^{\prime}(0) \\
(\mathbf{A D M}\end{array}$ \\
\hline 1.0 & 0.5278 & 0.5277 & 0.5279 & 0.9009 & 0.9008 & 0.9009 & 0.1170 & 0.1171 & 0.1169 & 0.2976 & 0.2975 & 0.2977 \\
\hline 2.0 & 0.5257 & 0.5256 & 0.5258 & 0.9014 & 0.9013 & 0.9014 & 0.1057 & 0.1058 & 0.1056 & 0.2968 & 0.2967 & 0.2969 \\
\hline 4.0 & 0.5233 & 0.5232 & 0.5234 & 0.9016 & 0.9015 & 0.9016 & 0.0805 & 0.0806 & 0.0804 & 0.2962 & 0.2961 & 0.2964 \\
\hline 8.0 & 0.5210 & 0.5208 & 0.5211 & 0.9017 & 0.9016 & 0.9018 & 0.0548 & 0.0549 & 0.0547 & 0.2960 & 0.2959 & 0.2961 \\
\hline 16.0 & 0.5192 & 0.5191 & 0.5193 & 0.9016 & 0.9015 & 0.9014 & 0.0343 & 0.0344 & 0.0342 & 0.2959 & 0.2958 & 0.2961 \\
\hline
\end{tabular}

Table 5: Comparison of RKM, HAM and ADM numerical solutions for various Prandtl numbers $(P r)$ with $M=3.0, N_{l}=0.2, G r=5.0, G=2.0, E c=0.3, m=1.0, n=1.0, \gamma=0.02$ and $\lambda=1.0$.

\begin{tabular}{|c|c|c|c|c|c|c|c|c|c|c|c|c|}
\hline $\operatorname{Pr}$ & $\begin{array}{c}f^{\prime \prime}(0) \\
(\mathbf{R K M})\end{array}$ & $\begin{array}{c}f^{\prime \prime}(0) \\
(\mathbf{H A M})\end{array}$ & $\begin{array}{c}f^{\prime \prime}(0) \\
(\mathbf{A D M})\end{array}$ & $\begin{array}{c}g^{\prime}(0) \\
(\mathbf{R K M})\end{array}$ & $\begin{array}{c}g^{\prime}(0) \\
(\mathbf{H A M})\end{array}$ & $\begin{array}{c}g^{\prime}(0) \\
(\mathbf{A D M})\end{array}$ & $\begin{array}{c}h^{\prime}(0) \\
(\mathbf{R K M})\end{array}$ & $\begin{array}{c}h^{\prime}(0) \\
(\mathbf{H A M})\end{array}$ & $\begin{array}{c}h^{\prime}(0) \\
(\mathbf{A D M})\end{array}$ & $\begin{array}{c}-\theta^{\prime}(0) \\
(\mathbf{R K M})\end{array}$ & $\begin{array}{c}-\theta^{\prime}(0) \\
(\mathbf{H A M})\end{array}$ & $\begin{array}{c}-\theta^{\prime}(0) \\
(\mathbf{A D M}\end{array}$ \\
\hline 0.72 & 0.5257 & 0.5256 & 0.5258 & 0.9014 & 0.9013 & 0.9015 & 0.1057 & 0.1056 & 0.1058 & 0.2968 & 0.2969 & 0.2967 \\
\hline 2.0 & 0.1992 & 0.1991 & 0.1993 & 0.7675 & 0.7673 & 0.7677 & 0.1836 & 0.1835 & 0.1838 & 0.5093 & 0.5092 & 0.5091 \\
\hline 3.7 & -0.0241 & -0.0240 & -0.0242 & 0.6949 & 0.6947 & 0.6950 & 0.2218 & 0.2217 & 0.2220 & 0.6879 & 0.6881 & 0.6878 \\
\hline 7.0 & -0.2519 & -0.2518 & -0.2520 & 0.6346 & 0.6344 & 0.6348 & 0.2516 & 0.2515 & 0.2518 & 0.9095 & 0.9097 & 0.9094 \\
\hline 10.0 & -0.3694 & -0.3693 & -0.3695 & 0.6087 & 0.6085 & 0.6088 & 0.2639 & 0.2640 & 0.2641 & 1.0431 & 1.0430 & 1.0430 \\
\hline
\end{tabular}

Table 6: Comparison of RKM, HAM and ADM numerical solutions for various Eckert numbers $(E c)$ with $M=3.0, N_{l}=0.2, G r=5.0, G=2.0, \operatorname{Pr}=0.72, m=1.0, n=1.0, \gamma=0.02$ and $\lambda=1.0$.

\begin{tabular}{|c|c|c|c|c|c|c|c|c|c|c|c|c|}
\hline$E c$ & $\begin{array}{c}f^{\prime \prime}(0) \\
(\mathbf{R K M})\end{array}$ & $\begin{array}{c}f^{\prime \prime}(0) \\
(\mathbf{H A M})\end{array}$ & $\begin{array}{c}f^{\prime \prime}(0) \\
(\mathbf{A D M})\end{array}$ & $\begin{array}{c}g^{\prime}(0) \\
(\mathbf{R K M}\end{array}$ & $\begin{array}{c}g^{\prime}(0) \\
(\mathbf{H A M}\end{array}$ & $\begin{array}{c}g^{\prime}(0) \\
(\mathbf{A D M})\end{array}$ & $\begin{array}{c}h^{\prime}(0) \\
(\mathbf{R K M})\end{array}$ & $\begin{array}{c}h^{\prime}(0) \\
(\mathbf{H A M})\end{array}$ & $\begin{array}{c}h^{\prime}(0) \\
(\mathbf{A D M})\end{array}$ & $\begin{array}{c}-\theta^{\prime}(0) \\
(\mathbf{R K M})\end{array}$ & $\begin{array}{c}-\theta^{\prime}(0) \\
(\mathbf{H A M})\end{array}$ & $\begin{array}{c}-\theta^{\prime}(0) \\
(\mathbf{A D M})\end{array}$ \\
\hline 0.003 & 0.3261 & 0.3262 & 0.3259 & 0.8460 & 0.8459 & 0.8461 & 0.1388 & 0.1389 & 0.1387 & 0.6066 & 0.6065 & 0.6068 \\
\hline 0.3 & 0.5257 & 0.5258 & 0.5256 & 0.9014 & 0.9013 & 0.9015 & 0.1057 & 0.1058 & 0.1056 & 0.2968 & 0.2967 & 0.2969 \\
\hline 1.0 & 1.5533 & 1.5534 & 1.5532 & 1.1245 & 1.1244 & 1.1246 & -0.0436 & -0.0437 & -0.0435 & -1.2828 & -1.2827 & -1.2829 \\
\hline 2.0 & 5.3200 & 5.3201 & 5.3199 & 1.5760 & 1.5759 & 1.5761 & -0.4029 & -0.4030 & -0.4028 & -9.1118 & -9.1117 & -9.1119 \\
\hline 2.5 & 8.0972 & 8.0973 & 8.0971 & 1.7896 & 1.7895 & 1.7897 & -0.5917 & -0.5918 & -0.5916 & -16.7787 & -16.7790 & -16.7789 \\
\hline
\end{tabular}

Table 7: Comparison of RKM, HAM and ADM numerical solutions for various Hall current parameters $(m)$ with $M=3.0, N_{l}=0.2, G r=5.0, G=2.0, \operatorname{Pr}=0.72, E c=0.3, n=1.0, \gamma=0.02$ and $\lambda=1.0$.

\begin{tabular}{|c|c|c|c|c|c|c|c|c|c|c|c|c|}
\hline$m$ & $\begin{array}{c}f^{\prime \prime}(0) \\
(\mathbf{R K M}\end{array}$ & $\begin{array}{c}f^{\prime \prime}(0) \\
\mathbf{( H A M})\end{array}$ & $\begin{array}{c}f^{\prime \prime}(0) \\
(\mathbf{A D M})\end{array}$ & $\begin{array}{c}g^{\prime}(0) \\
(\mathbf{R K M})\end{array}$ & $\begin{array}{c}g^{\prime}(0) \\
(\mathbf{H A M})\end{array}$ & $\begin{array}{c}g^{\prime}(0) \\
(\mathbf{A D M})\end{array}$ & $\begin{array}{c}h^{\prime}(0) \\
(\mathbf{R K M})\end{array}$ & $\begin{array}{c}h^{\prime}(0) \\
(\mathbf{H A M})\end{array}$ & $\begin{array}{c}h^{\prime}(0) \\
(\mathbf{A D M})\end{array}$ & $\begin{array}{c}-\theta^{\prime}(0) \\
(\mathbf{R K M})\end{array}$ & $\begin{array}{c}-\theta^{\prime}(0) \\
(\mathbf{H A M})\end{array}$ & $\begin{array}{c}-\theta^{\prime}(0) \\
\text { (ADM }\end{array}$ \\
\hline 0.2 & 0.1709 & 0.1710 & 0.1708 & 0.2789 & 0.2788 & 0.2788 & 0.1182 & 0.1183 & 0.1182 & 0.0546 & 0.0544 & 0.0547 \\
\hline 0.5 & 0.2814 & 0.2815 & 0.2813 & 0.6200 & 0.6201 & 0.6219 & 0.1152 & 0.1151 & 0.1150 & 0.1310 & 0.1311 & 0.1311 \\
\hline 1.0 & 0.5257 & 0.5258 & 0.5256 & 0.9014 & 0.9015 & 0.9013 & 0.1057 & 0.1058 & 0.1056 & 0.2968 & 0.2969 & 0.2969 \\
\hline 1.5 & 0.7272 & 0.7273 & 0.7271 & 0.9437 & 0.9438 & 0.9436 & 0.0942 & 0.0943 & 0.0941 & 0.4263 & 0.4264 & 0.4264 \\
\hline 2.0 & 0.8679 & 0.8680 & 0.8678 & 0.8918 & 0.8919 & 0.8917 & 0.0838 & 0.0839 & 0.0837 & 0.5102 & 0.5103 & 0.5104 \\
\hline
\end{tabular}


Table 8: Comparison of RKM, HAM and ADM numerical solutions for various wall distention parameters $(n)$ with $M=3.0, N_{1}=0.2, G r=5.0, G=2.0, P r=0.72, E c=0.3, m=1.0, \gamma=0.02$ and $\lambda=1.0$.

\begin{tabular}{|c|c|c|c|c|c|c|c|c|c|c|c|c|}
\hline$n$ & $\begin{array}{c}f^{\prime \prime}(0) \\
(\mathbf{R K M})\end{array}$ & $\begin{array}{c}f^{\prime \prime}(0) \\
(\mathbf{H A M})\end{array}$ & $\begin{array}{c}f^{\prime \prime}(0) \\
\mathbf{( A D M})\end{array}$ & $\begin{array}{c}g^{\prime}(0) \\
(\mathbf{R K M})\end{array}$ & $\begin{array}{c}g^{\prime}(0) \\
(\mathbf{H A M}\end{array}$ & $\begin{array}{c}g^{\prime}(0) \\
(\mathbf{A D M}\end{array}$ & $\begin{array}{c}h^{\prime}(0) \\
(\mathbf{R K M})\end{array}$ & $\begin{array}{c}h^{\prime}(0) \\
(\mathbf{H A M})\end{array}$ & $\begin{array}{c}h^{\prime}(0) \\
(\mathbf{A D M})\end{array}$ & $\begin{array}{c}-\theta^{\prime}(0) \\
(\mathbf{R K M})\end{array}$ & $\begin{array}{c}-\theta^{\prime}(0) \\
(\mathbf{H A M})\end{array}$ & $\begin{array}{c}-\theta^{\prime}(0) \\
(\mathbf{A D M}\end{array}$ \\
\hline-0.6 & 3.1897 & 3.1896 & 3.1898 & 1.8043 & 1.8042 & 1.8044 & -0.0867 & -0.0866 & -0.0868 & 2.1437 & 2.1438 & 2.1436 \\
\hline-0.3 & 1.7510 & 1.7511 & 1.7512 & 1.1183 & 1.1181 & 1.1184 & 0.1401 & 0.1400 & 0.1402 & 1.6947 & 1.6949 & 1.6946 \\
\hline 0.0 & 1.0560 & 1.0561 & 1.0562 & 0.9539 & 0.9538 & 0.9537 & 0.1840 & 0.1839 & 0.1841 & 1.4433 & 1.4432 & 1.4432 \\
\hline 0.3 & 0.6427 & 0.6428 & 0.6425 & 0.8761 & 0.8760 & 0.8762 & 0.1888 & 0.1887 & 0.1889 & 1.2839 & 1.2838 & 1.2838 \\
\hline 0.6 & 0.3650 & 0.3651 & 0.3652 & 0.8292 & 0.8291 & 0.8293 & 0.1830 & 0.1829 & 0.1831 & 1.1724 & 1.1725 & 1.1723 \\
\hline 1.0 & 0.1084 & 0.1085 & 0.1082 & 0.7887 & 0.7886 & 0.7888 & 0.1712 & 0.1711 & 0.1713 & 1.0663 & 1.0664 & 1.0662 \\
\hline 2.0 & -0.2659 & -0.2658 & -0.2658 & 0.7325 & 0.7324 & 0.7326 & 0.1427 & 0.1426 & 0.1428 & 0.9062 & 0.9063 & 0.9061 \\
\hline 3.0 & -0.9062 & -0.9061 & -0.9061 & 0.7018 & 0.7017 & 0.7019 & 0.1213 & 0.1212 & 0.1214 & 0.8151 & 0.8152 & 0.8150 \\
\hline
\end{tabular}

Table 9: Comparison of RKM, HAM and ADM numerical solutions for various non-isothermal power-law index parameters $(\gamma)$ with $M=3.0, N_{1}=0.2, G r=5.0, G=2.0, P r=0.72, E c=0.3, m=1.0, n=1.0$ and $\lambda=1.0$.

\begin{tabular}{|c|c|c|c|c|c|c|c|c|c|c|c|c|}
\hline$n$ & $\begin{array}{c}f^{\prime \prime}(0) \\
(\mathbf{R K M})\end{array}$ & $\begin{array}{c}f^{\prime \prime}(0) \\
(\mathbf{H A M})\end{array}$ & $\begin{array}{c}f^{\prime \prime}(0) \\
(\mathbf{A D M})\end{array}$ & $\begin{array}{c}g^{\prime}(0) \\
(\mathbf{R K M})\end{array}$ & $\begin{array}{c}g^{\prime}(0) \\
(\mathbf{H A M})\end{array}$ & $\begin{array}{c}g^{\prime}(0) \\
(\mathbf{A D M}\end{array}$ & $\begin{array}{c}h^{\prime}(0) \\
(\mathbf{R K M})\end{array}$ & $\begin{array}{c}h^{\prime}(0) \\
(\mathbf{H A M})\end{array}$ & $\begin{array}{c}h^{\prime}(0) \\
(\mathbf{A D M})\end{array}$ & $\begin{array}{c}-\theta^{\prime}(0) \\
(\mathbf{R K M})\end{array}$ & $\begin{array}{c}-\theta^{\prime}(0) \\
(\mathbf{H A M})\end{array}$ & $\begin{array}{c}-\theta^{\prime}(0) \\
\text { (ADM) }\end{array}$ \\
\hline-1.0 & 1.2266 & 1.2267 & 1.2265 & 1.0599 & 1.0598 & 1.0597 & 0.0024 & 0.0023 & 0.0022 & -0.8039 & -0.8040 & -0.8041 \\
\hline-0.5 & 0.7707 & 0.7708 & 0.7706 & 0.9615 & 0.9616 & 0.9614 & 0.0680 & 0.0681 & 0.0682 & -0.0953 & -0.0952 & -0.0954 \\
\hline 0.0 & 0.5331 & 0.5332 & 0.5330 & 0.9033 & 0.9035 & 0.9032 & 0.1045 & 0.1046 & 0.1047 & 0.2847 & 0.2846 & 0.2849 \\
\hline 0.5 & 0.3791 & 0.3792 & 0.3790 & 0.8630 & 0.8631 & 0.8629 & 0.1287 & 0.1288 & 0.1288 & 0.5461 & 0.5460 & 0.5463 \\
\hline 1.0 & 0.2672 & 0.2673 & 0.2671 & 0.8327 & 0.8328 & 0.8326 & 0.1464 & 0.1465 & 0.1463 & 0.7495 & 0.7494 & 0.7497 \\
\hline 2.0 & 0.1084 & 0.1085 & 0.1082 & 0.7887 & 0.7888 & 0.7886 & 0.1712 & 0.1713 & 0.1711 & 1.0663 & 1.065 & 1.068 \\
\hline
\end{tabular}

Table 10: Comparison between present results and previously published results for dimensionless skin friction, $f^{\prime \prime}(0)$.

\begin{tabular}{|c|c|c|c|}
\hline $\begin{array}{c}\text { Gorla and } \\
\text { Sidawi [53] }\end{array}$ & RKM & HAM & ADM \\
\hline-1.01435 & -1.01434 & -1.01436 & -1.01434 \\
\hline
\end{tabular}

\section{Numerical results and discussion}

In all graphical plots we have adopted RKM solutions. The maximum value of $\eta_{\infty}$ to each group of parameters $M, N_{1}, G r, m, G, \operatorname{Pr}, E c, \gamma$ and $n$ has been determined when the values of the unknown boundary conditions at $\eta=0$ do not change in the computational loop and an error less than $10^{-5}$ is achieved. In order to verify the effects of the step size $\Delta \eta$, we have run the code for our model with three different step sizes as $\Delta \eta=0.01, \Delta \eta=0.005$ and $\Delta \eta=0.001$, in each case we have found excellent agreement among them as shown in Figs 2 to 5. For the purpose of discussing the results of the flow field represented in the Fig 1, the numerical computations are presented in the form of non-dimensional primary velocity and secondary velocity, angular velocity (micro-rotation) and temperature functions. As demonstrated in the above model, the angle between the direction of the strong uniform magnetic field and the plane transverse to the plate is zero i.e., $\alpha=0^{\circ}$, hence the value of the parameter $\lambda$ is prescribed as unity. In a separate study the effect of oblique magnetic field is examined [54]. Extensive numerical computations have been conducted for variation of the other 9 
parameters arising in the mathematical model, namely magnetohydrodynamic body force parameter $(M)$, micropolar material parameter $\left(N_{1}\right)$, Grashof number $(G r)$, Hall parameter $(m)$ micro-rotational parameter $(G)$, Prandtl number $(\operatorname{Pr})$, Eckert number $(E c)$, non-isothermal power-law index $(\gamma)$ and non-linear wall geometric parameter $(n)$. The distributions for the primary velocity, secondary velocity, angular velocity (micro-element spin) and temperature functions are displayed in Figs. 6 - 41.

Figs. 6-9 illustrate the effects of magnetic field parameter $M . M$ has evidently a suppressive effect on primary velocity profiles as well as angular velocity profiles exposed in Figs. 6, 8. Micro-rotation and primary velocity are therefore strongly damped with greater positive magnetic field parameter $(M)$ owing to the Lorentzian drag force components which appear as respectively, $-\frac{2}{n+1}\left[\frac{M \lambda}{1+m^{2} \lambda^{2}}\left(f^{\prime}+m \lambda g\right)\right]$ and $-\frac{2}{n+1}\left[n f^{\prime} g-\frac{M \lambda}{1+m^{2} \lambda^{2}}\left(m \lambda f^{\prime}-g\right)\right]$ in the primary (12) and secondary momenta conservation equations (13). Close inspection of these forces reveals that the primary Lorentzian body force is negative and therefore impedes the flow, whereas the secondary Lorentzian body force is in fact positive and is assistive to secondary momentum development (when $M>0$ ). The strong coupling of the micro-rotation eqn. (14) to the primary momentum equation (12) via the $-f^{\prime \prime}$ term in the former and the $-N_{1} h^{\prime}$ term in the latter is responsible for the damping of the micro-rotation with magnetic field- it is an indirect effect transferred from the initial suppression in primary velocity (as observed in Fig 6) to the subsequent depletion in angular velocity (as seen in Fig. 8). Greater regulation (i.e. deceleration) of the primary flow and spin of micro-elements is therefore attained with stronger magnetic field. We note that despite the inhibiting effect of magnetic field on the primary flow, flow reversal is never induced (primary velocity remains positive even with very strong magnetic field). The classical velocity shoot near the generator wall is also captured in the computations (fig. 6). Overall the implications for MHD generator performance is that subtle adjustments can be achieved in performance via the modification in transverse magnetic field $\left(M=\sigma \mu_{e} B_{0}{ }^{2} x / \rho U\right)$ since $M$ is linearly directly proportional to $B_{0}$. Velocity values can be fed into formulae for flow rate and efficiency and then optimal systems designed. Asymptotically smooth solutions are obtained for all $M$ values as shown by the gradual descent of all profiles with large $\eta$ values, confirming the prescription of an adequately large infinity boundary condition. Figs 7 and 9 indicate that the secondary velocity (cross flow) is substantially accelerated with greater positive $M$ values and temperature is also enhanced significantly for positive $M$. However, with negative $\mathrm{M}$ the reverse trend is observed. The case of $M<0$ corresponds to a reversal in the direction of application of the magnetic field. Rather than being directed towards the plate, it is orientated away from the plate. This causes the boundary layer to be dragged and manifests in a retarding influence in the secondary flow i.e. $g$ (fig. 7) as opposed to an acceleration in the primary flow i.e. $f^{\prime}$ (fig. 6). The secondary flow is so impeded that backflow is induced as observed in fig. 7. The acceleration in the micro-rotation $(h)$ as witnessed in fig. 8 , is principally induced via the acceleration in the primary translational flow $\left(f^{\prime}\right)$. The enhancement in temperatures (fig. 9) with positive $M$ is attributable to the dissipation in kinetic energy expended in dragging the micropolar gas against the action of transverse magnetic field (for $M>0$ the field is inhibiting, for $M<0$ it is assistive). This supplementary work manifests in the addition of thermal energy to the gas which causes a rise in temperature. Another interesting feature of figs 6-9 is that peak primary velocity is always close to the wall (and migrates closer to it with greater magnetic field). 


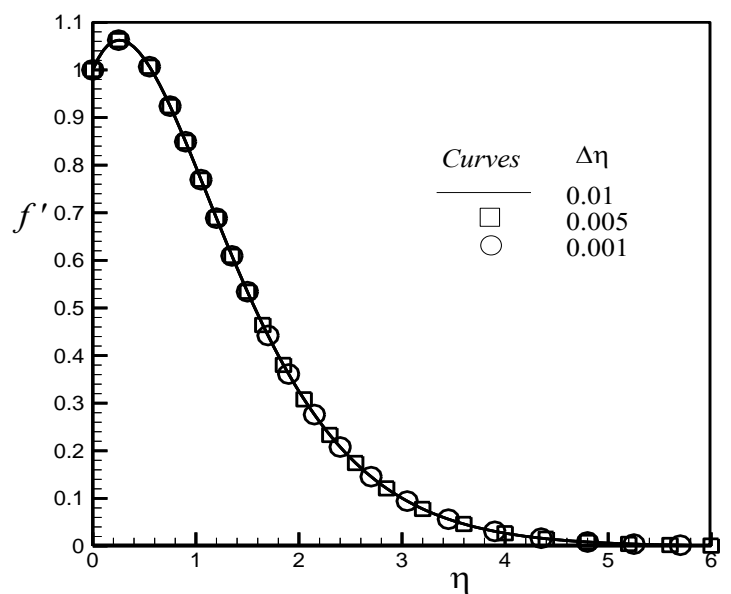

Fig. 2: Distribution of Primary velocity profiles for $\Delta \eta$

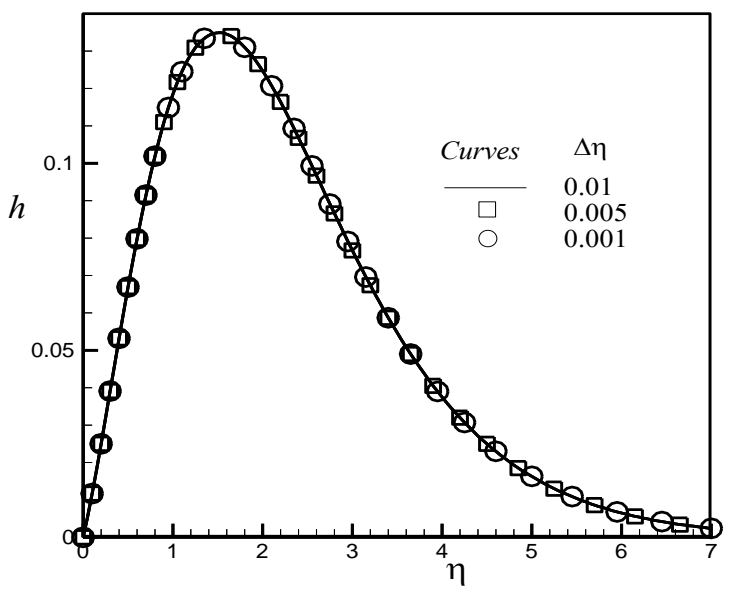

Fig. 4: Distribution of Microrotation profiles for $\Delta \eta$

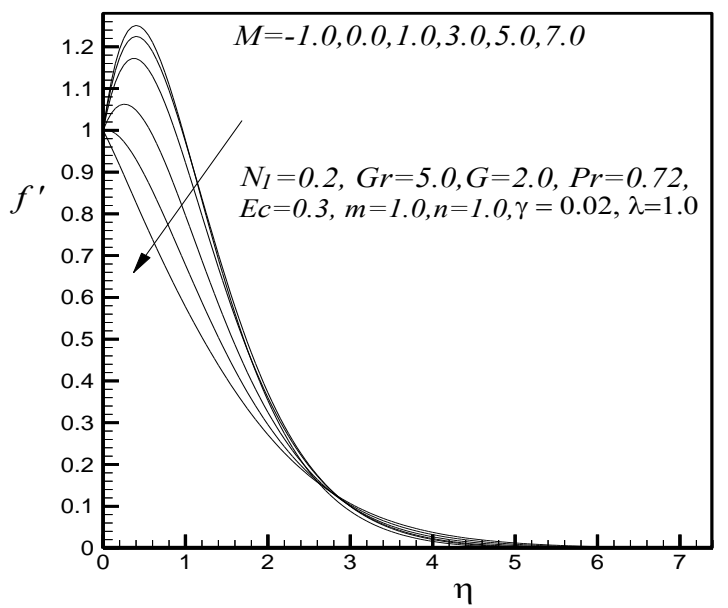

Fig. 6: Distribution of primary velocity profiles for $M$.

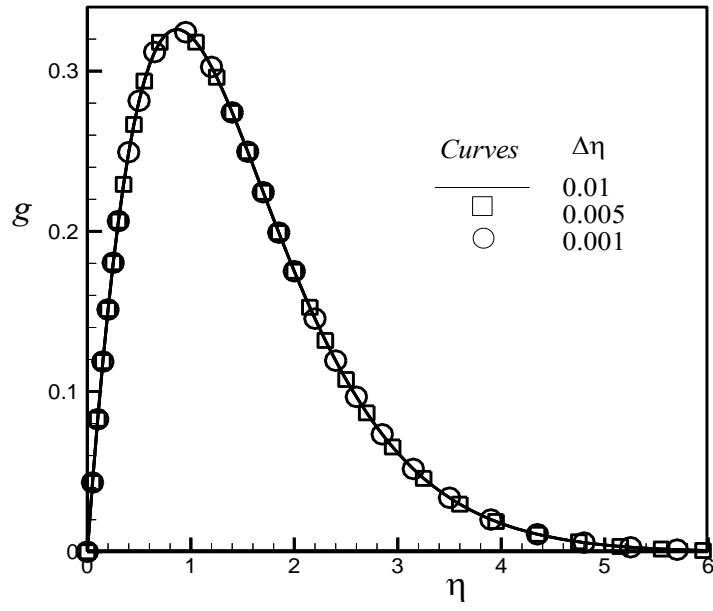

Fig. 3: Distribution of Secondary velocity profiles for $\Delta \eta$

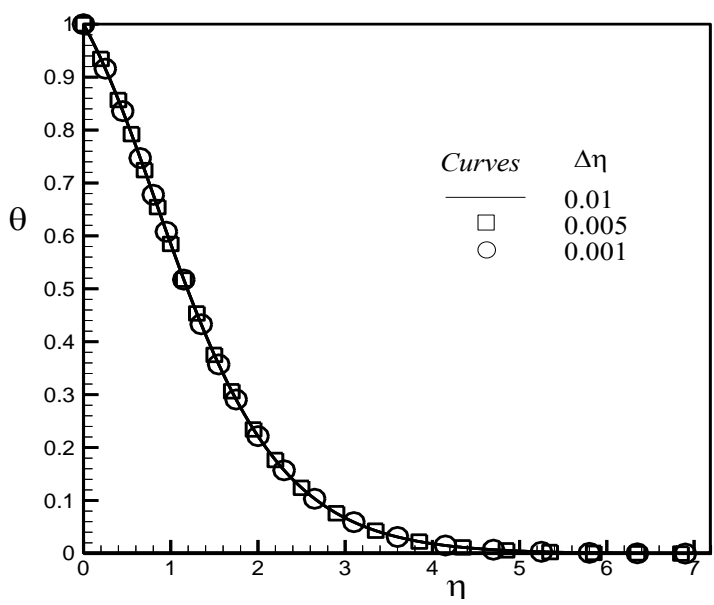

Fig. 5: Distribution of Temperature profiles for $\Delta \eta$

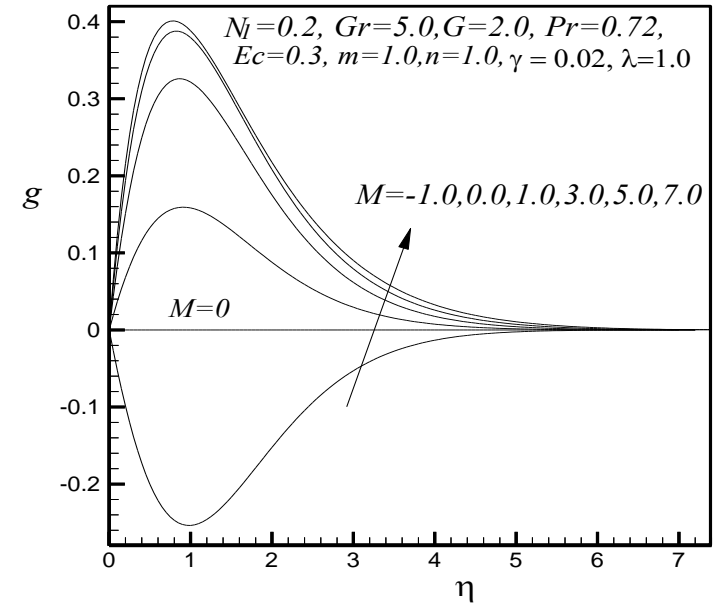

Fig. 7: Distribution of secondary velocity profiles for $M$. 


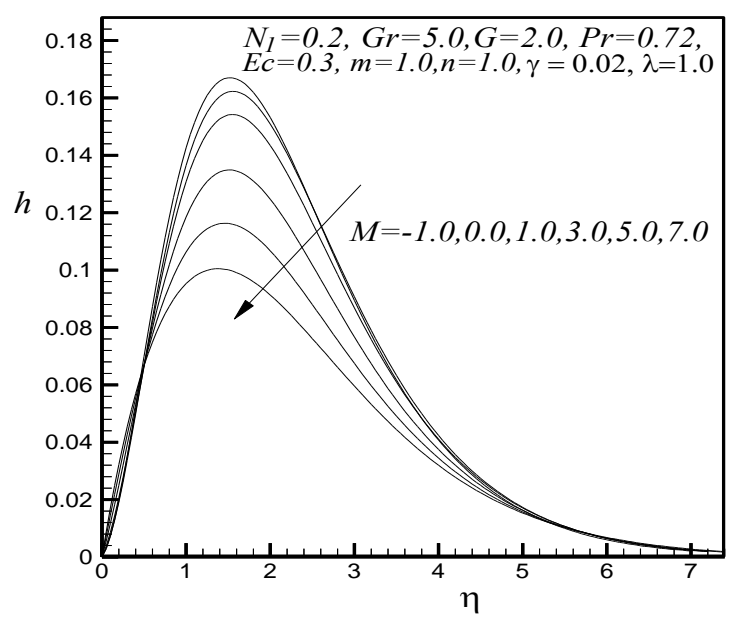

Fig. 8: Distribution of angular velocity profiles for $M$.

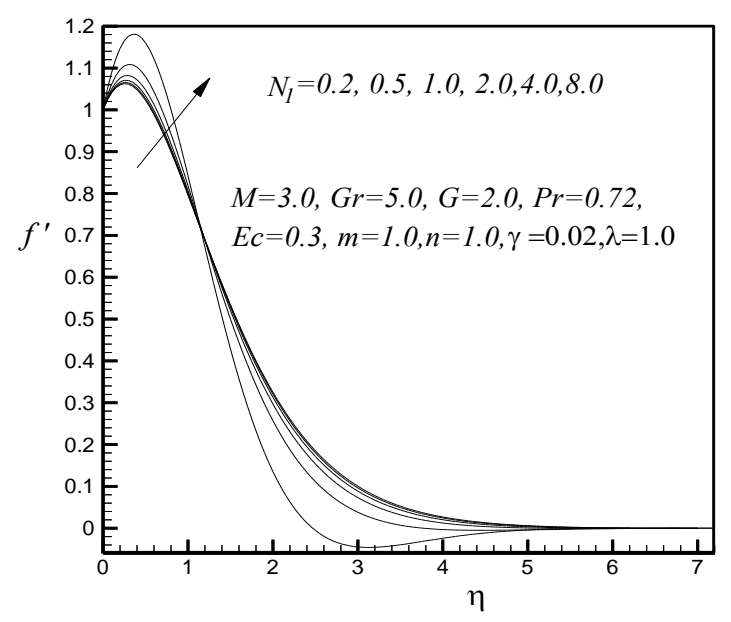

Fig. 10: Distribution of primary velocity profiles for $N_{1}$.

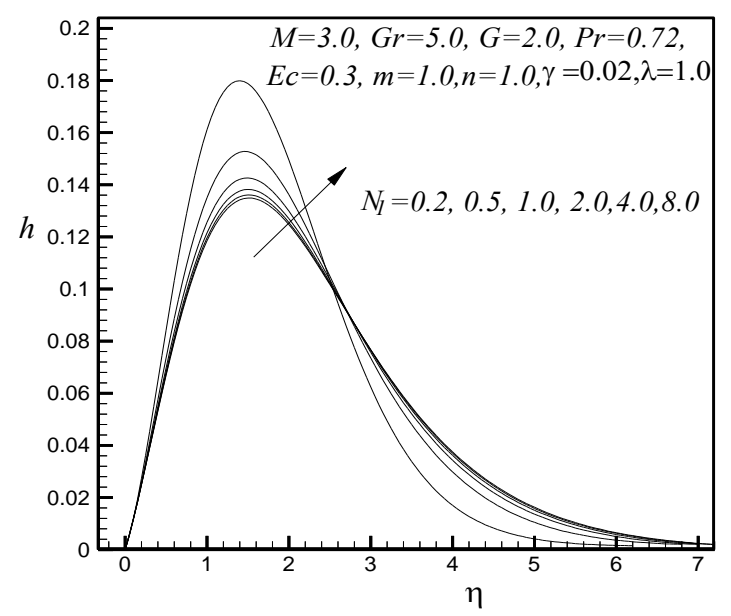

Fig. 12: Distribution of angular velocity for $N_{1}$.

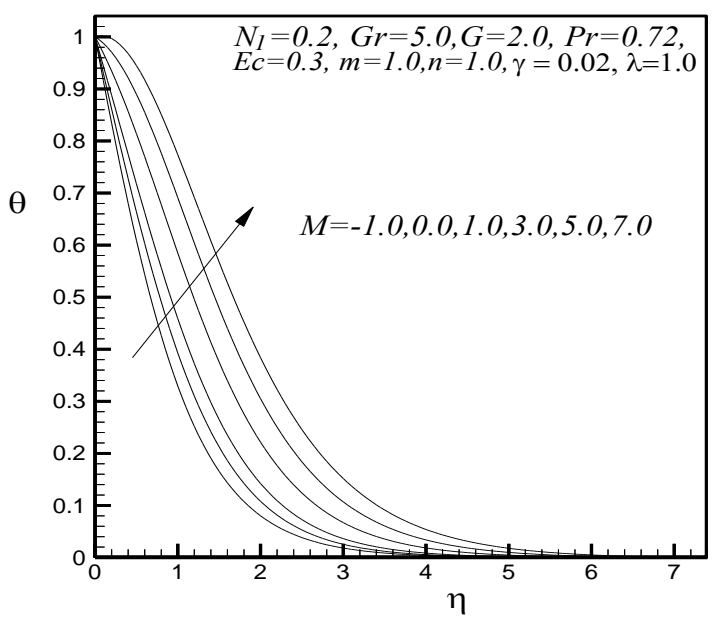

Fig. 9: Distribution of temperature profiles for M.

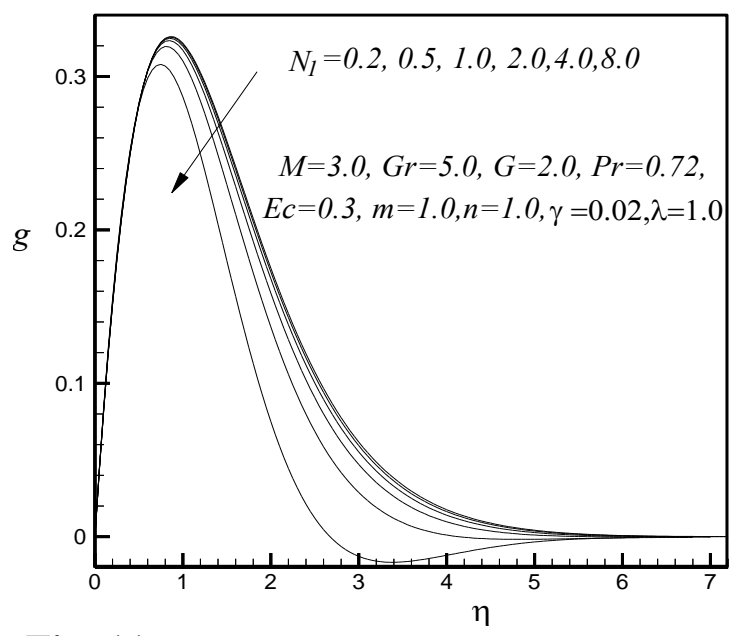

Fig. 11: Distribution of secondary velocity profiles for $N_{1}$.

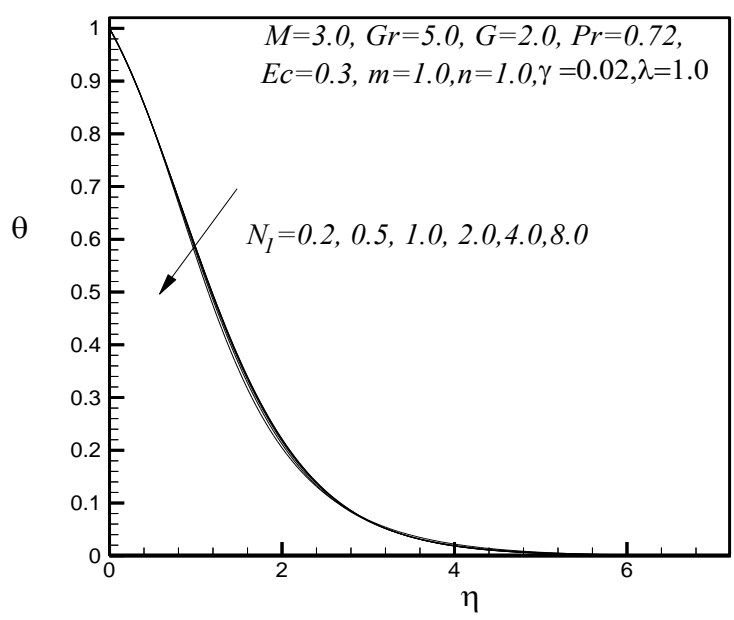

Fig. 13: Distribution of temperature for $N_{1}$. 


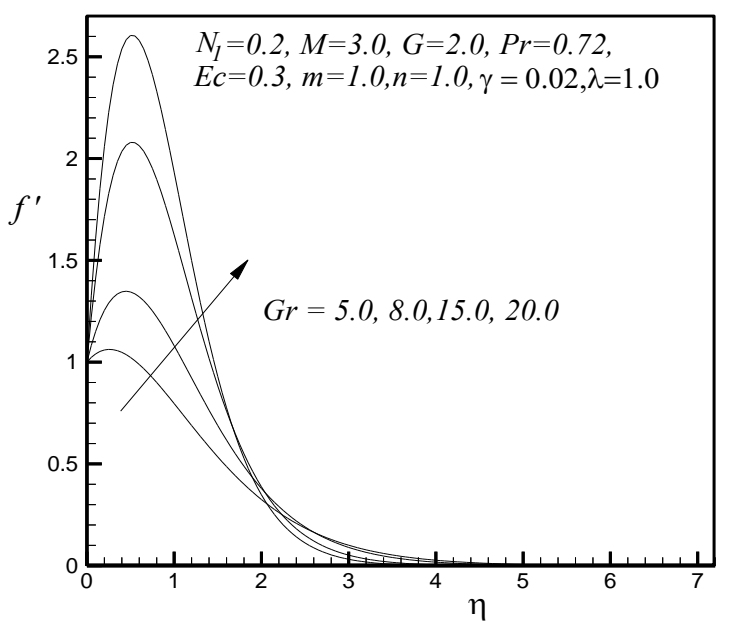

Fig. 14: Distribution of primary velocity profiles for $G r$.

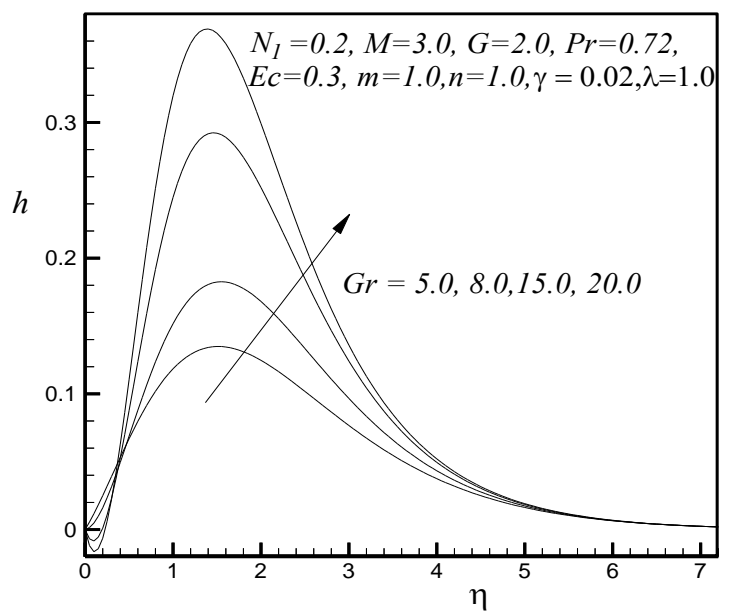

Fig. 16: Distribution of angular velocity profiles for $G r$.

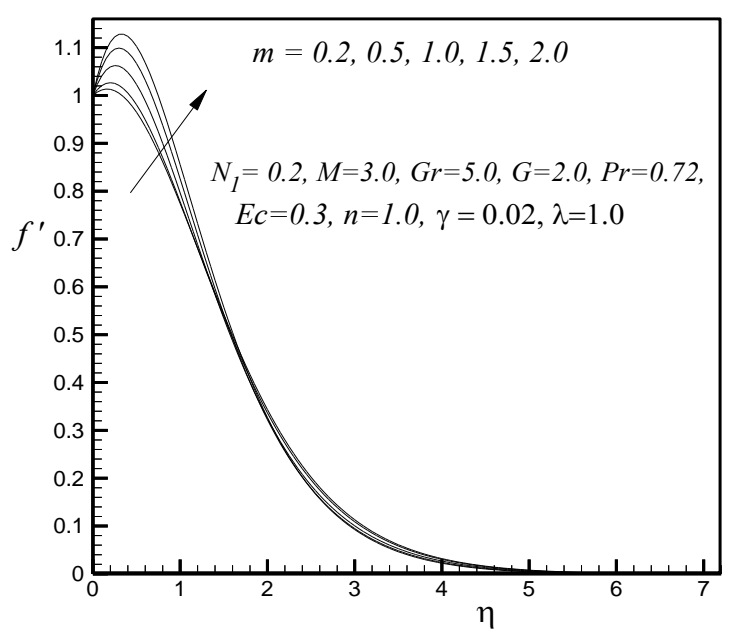

Fig. 18: Distribution of primary velocity for $m$.

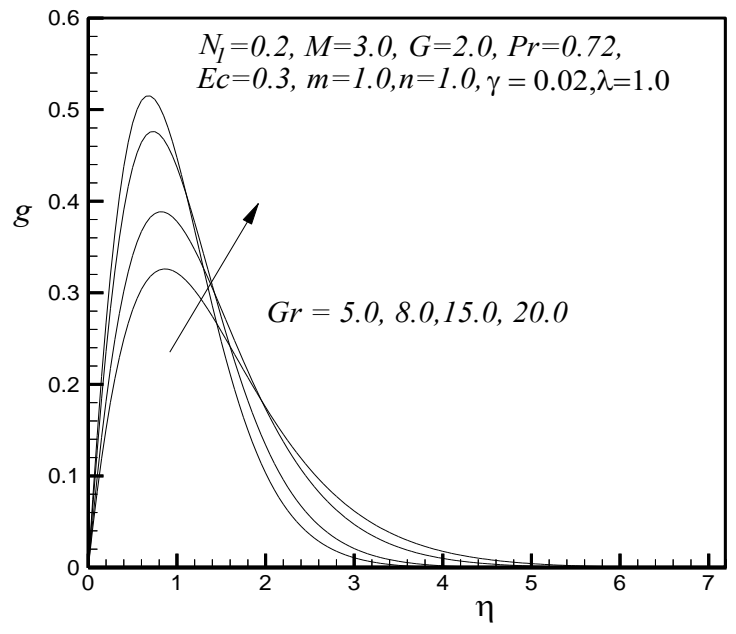

Fig. 15: Distribution of secondary velocity profiles for $G r$.

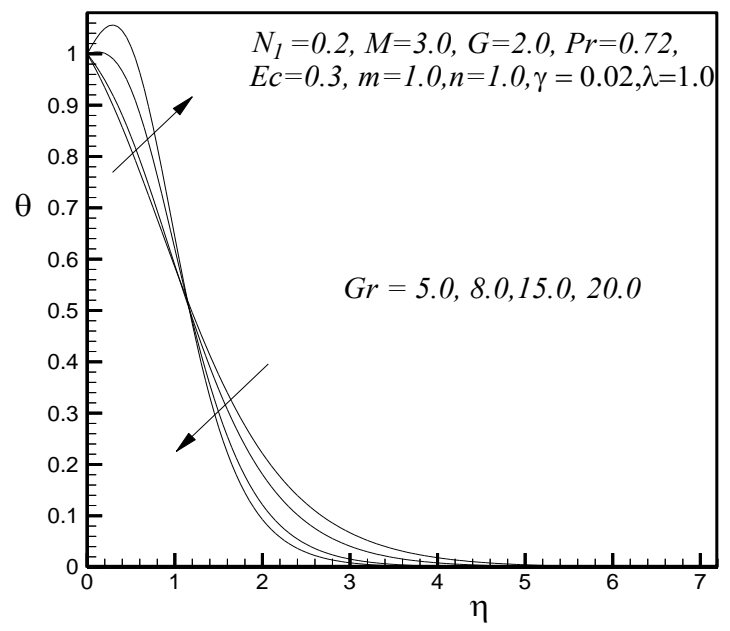

Fig. 17: Distribution of temperature for $G r$.

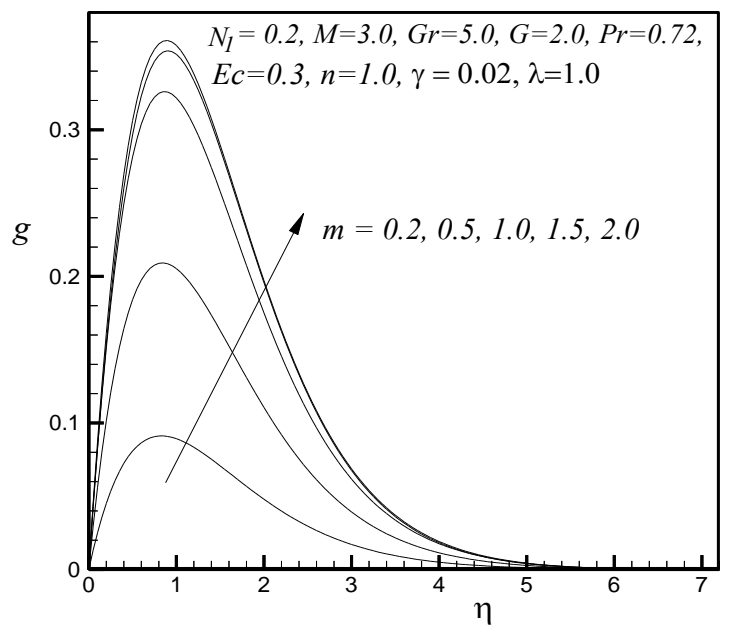

Fig. 19: Distribution of cross velocity for $m$. 


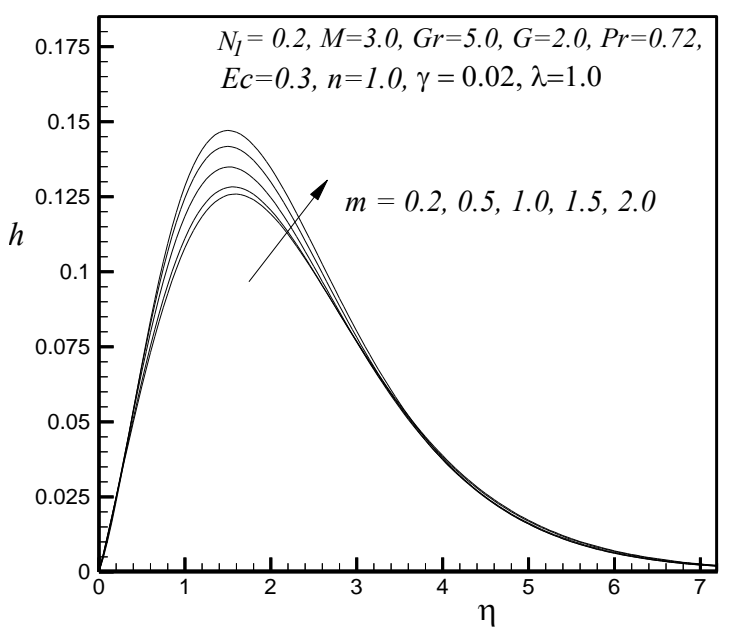

Fig. 20: Distribution of angular velocity for $m$.

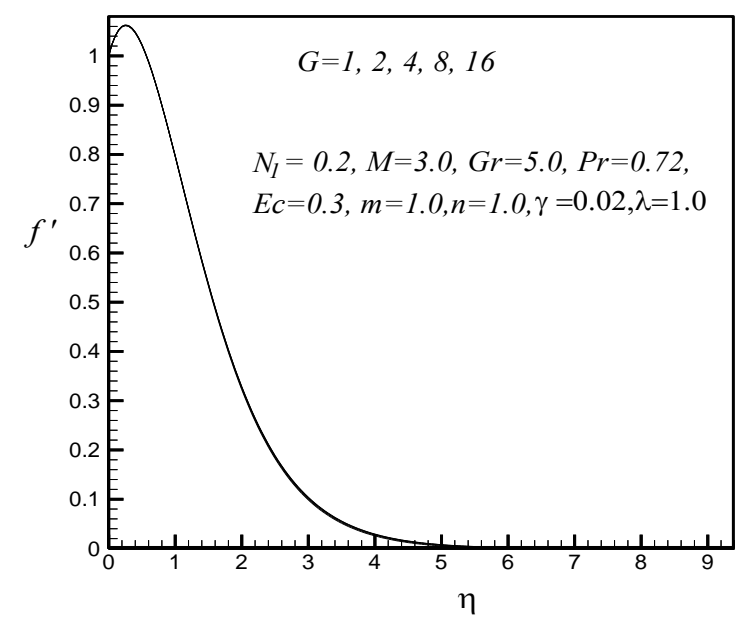

Fig. 22: Distribution of primary velocity for $G$.

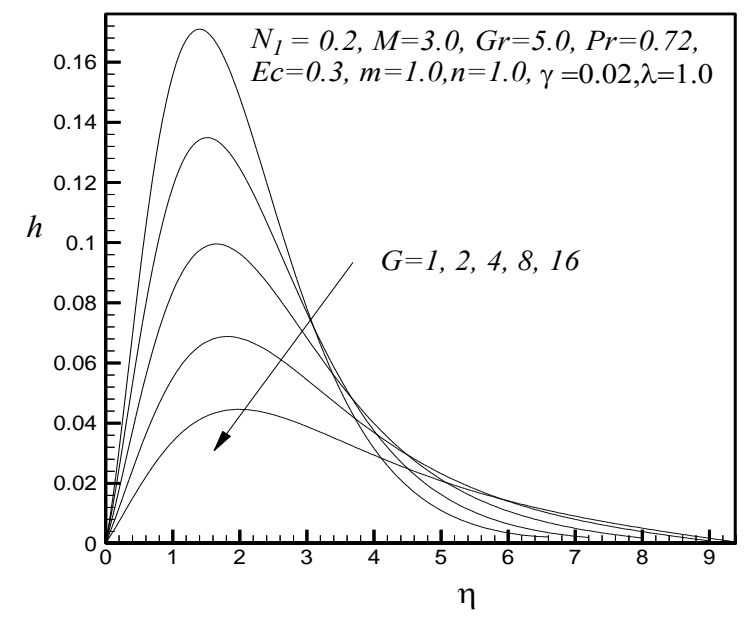

Fig. 24: Distribution of angular velocity profiles for $G$.

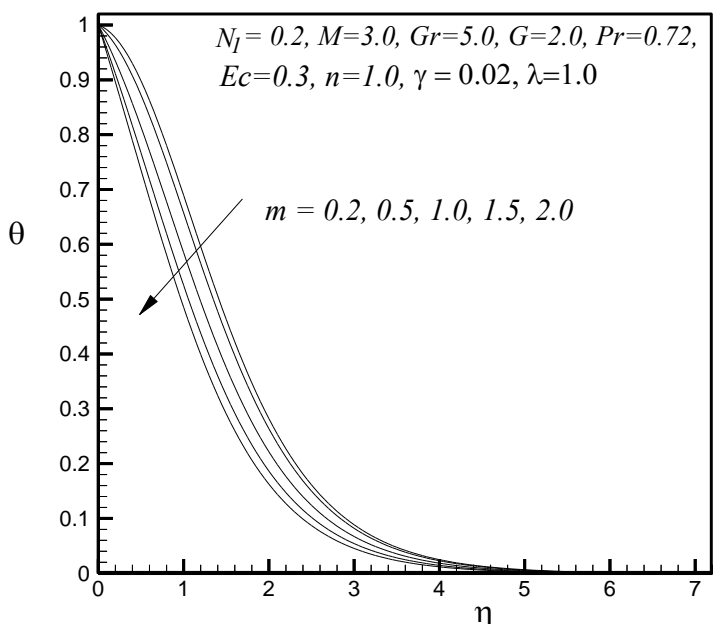

Fig. 21: Distribution of temperature for $m$.

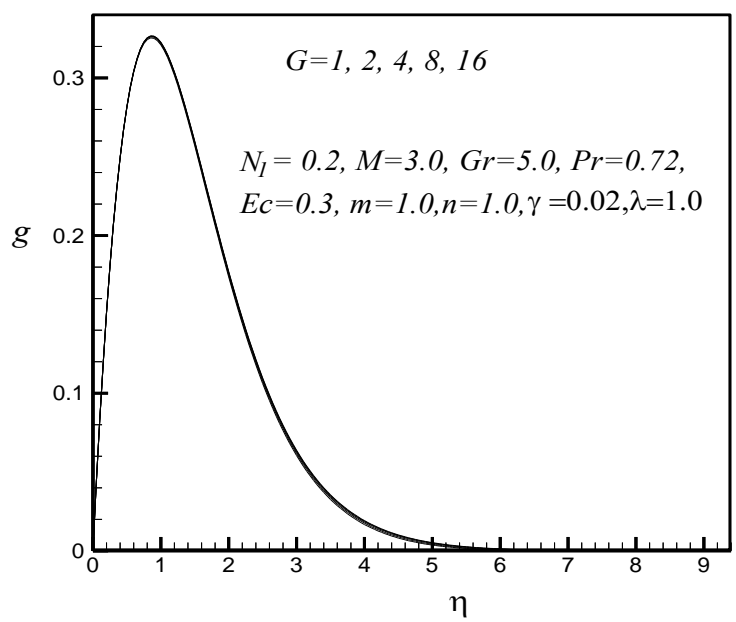

Fig. 23: Distribution of cross velocity for $G$.

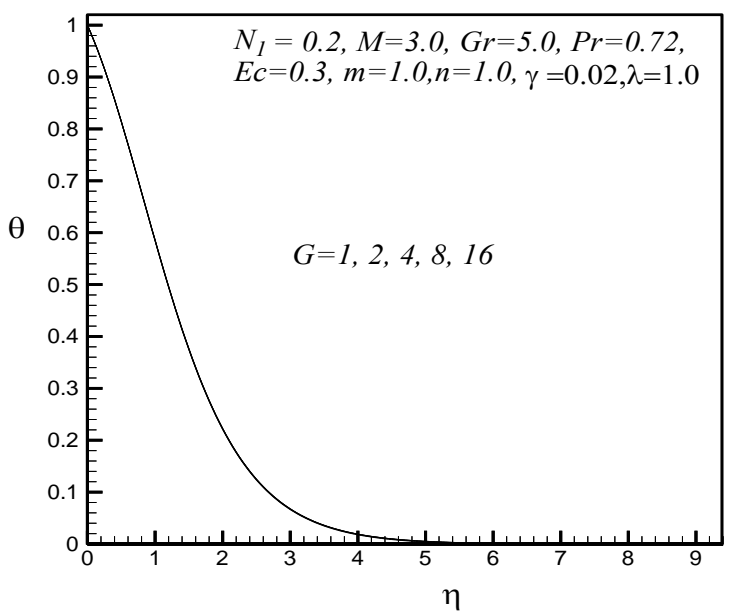

Fig. 25: Distribution of temperature profiles for $G$. 


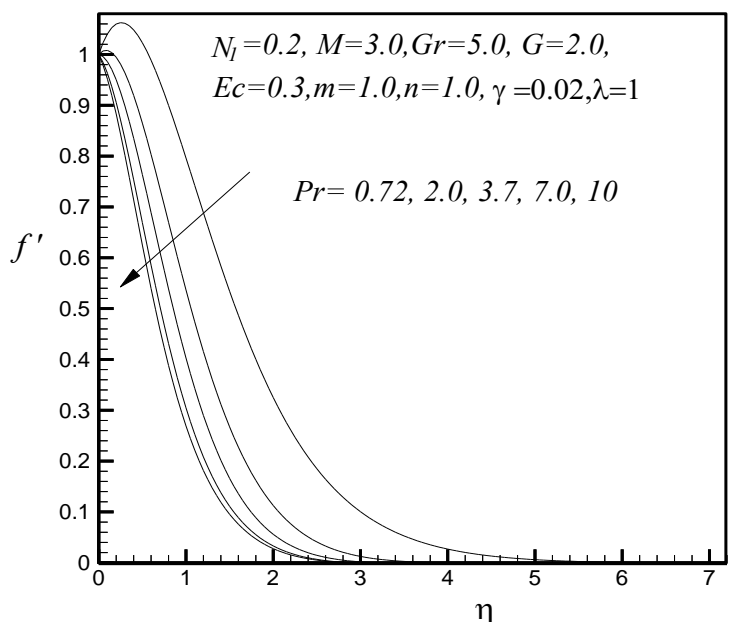

Fig. 26: Distribution of primary velocity profiles for Pr.

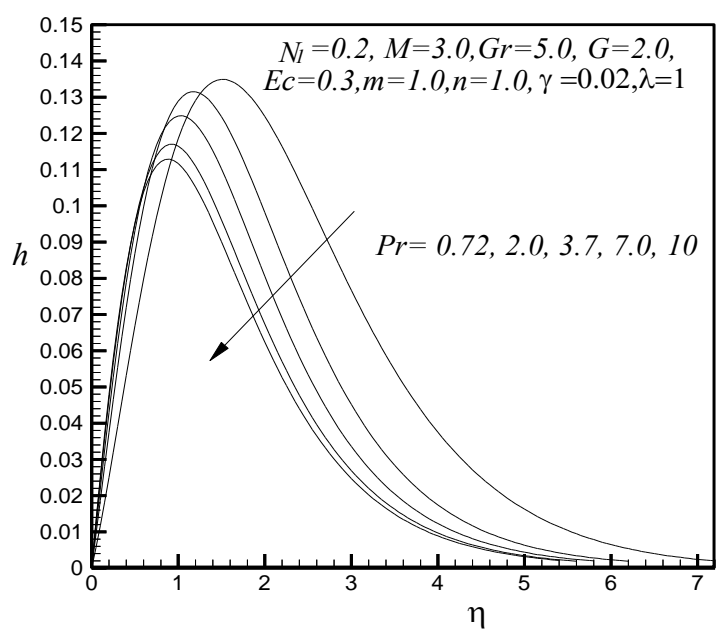

Fig. 28: Distribution of angular velocity for $\mathrm{Pr}$.

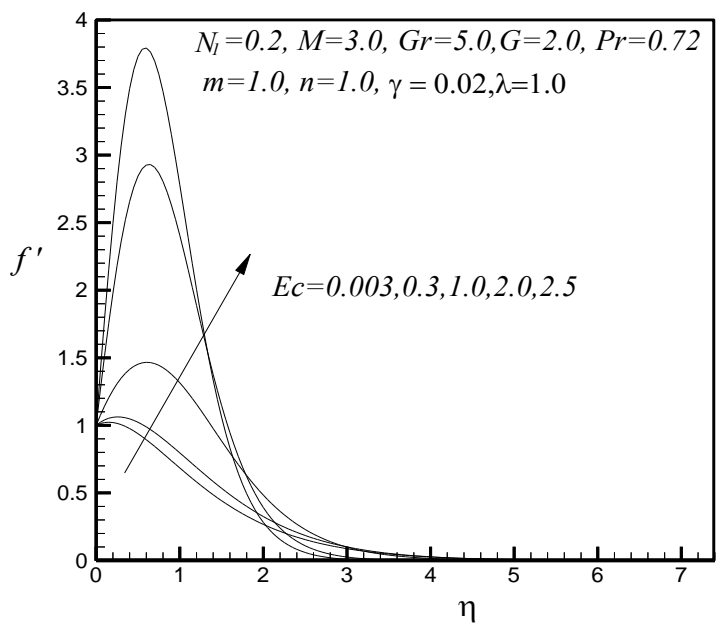

Fig. 30: Distribution of primary velocity for Ec.

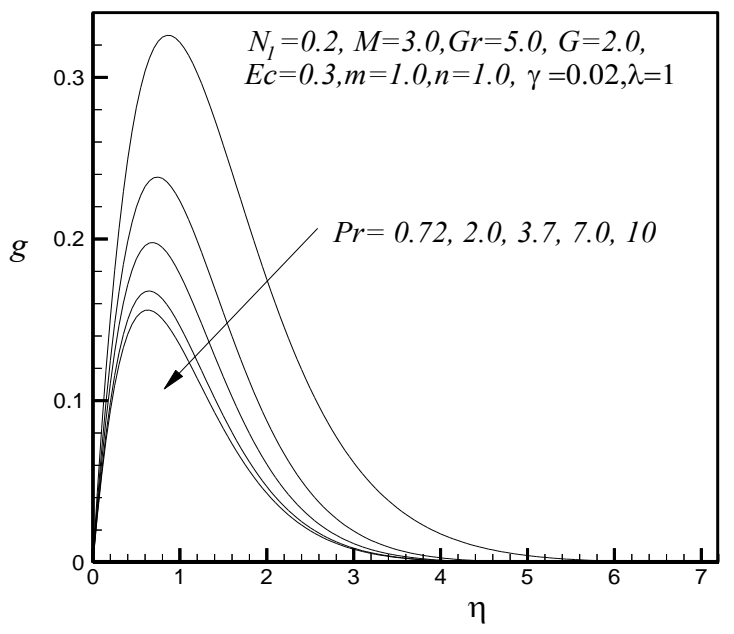

Fig. 27: Distribution of secondary velocity profiles for Pr.

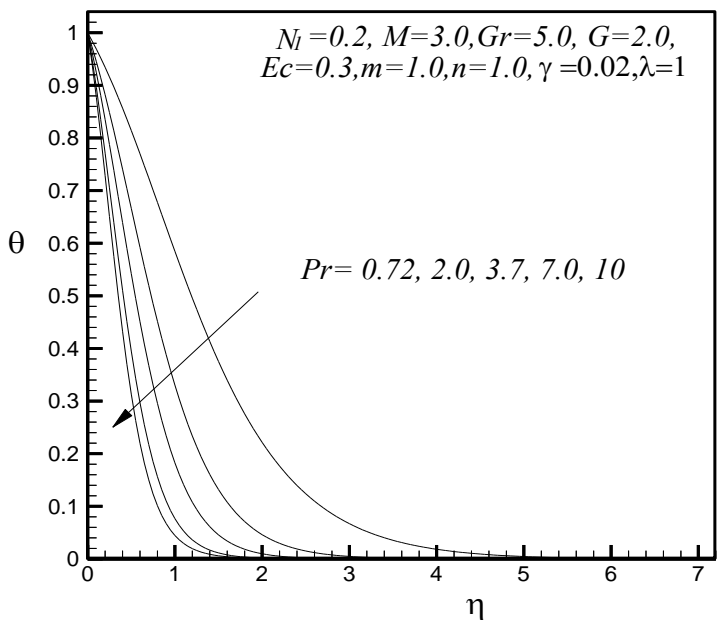

Fig. 29: Distribution of temperature for Pr.

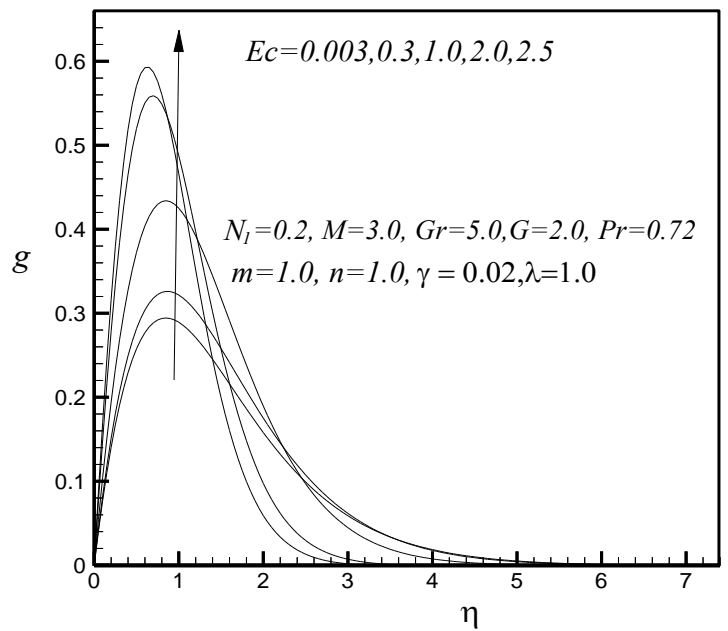

Fig. 31: Distribution of secondary velocity for Ec. 


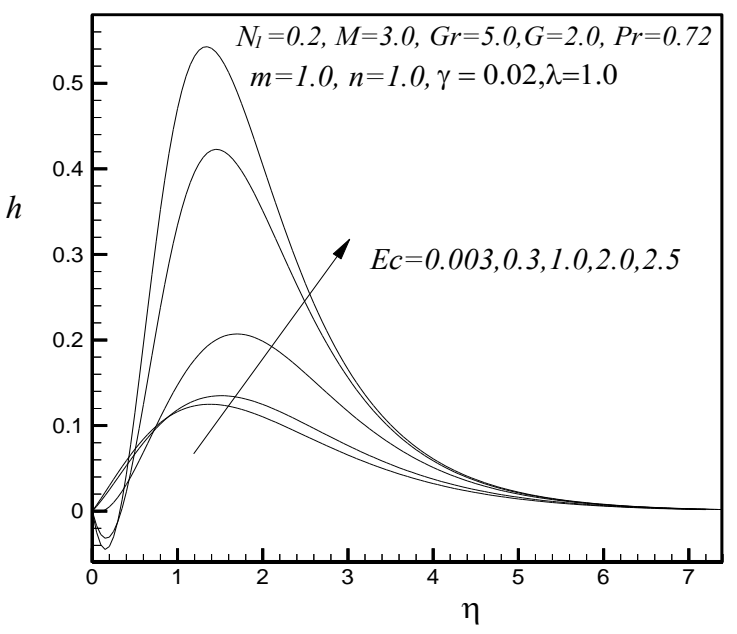

Fig. 32: Distribution of angular velocity profiles for $E c$.

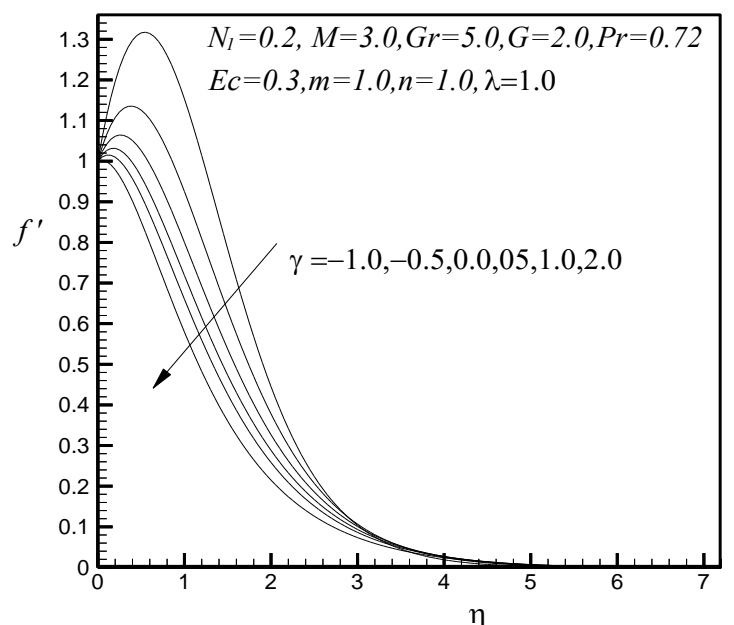

Fig. 34: Distribution of primary velocity profiles for $\gamma$.

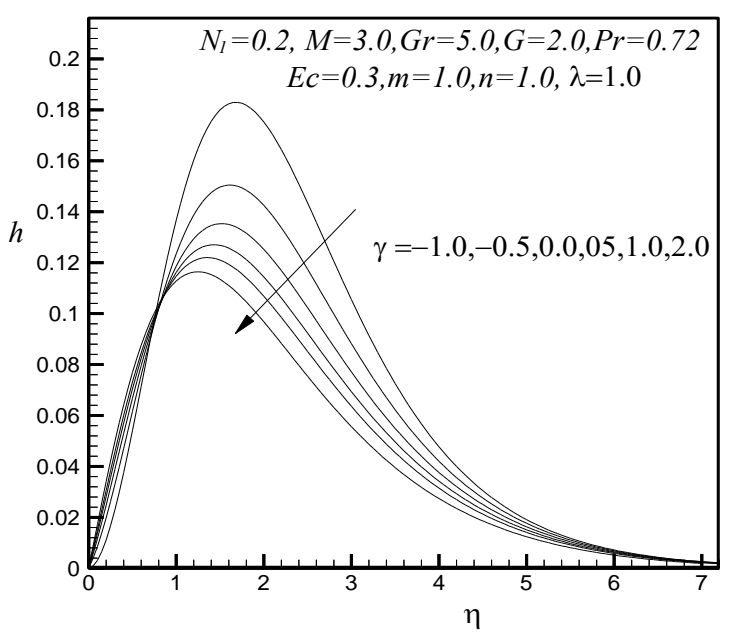

Fig. 36: Distribution of angular velocity profiles for $\gamma$.

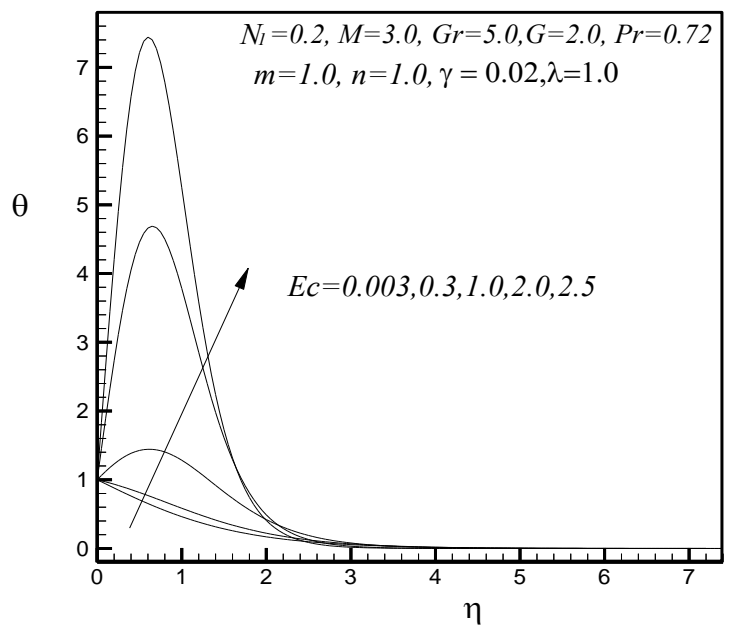

Fig. 33: Distribution of temperature profiles for Ec.

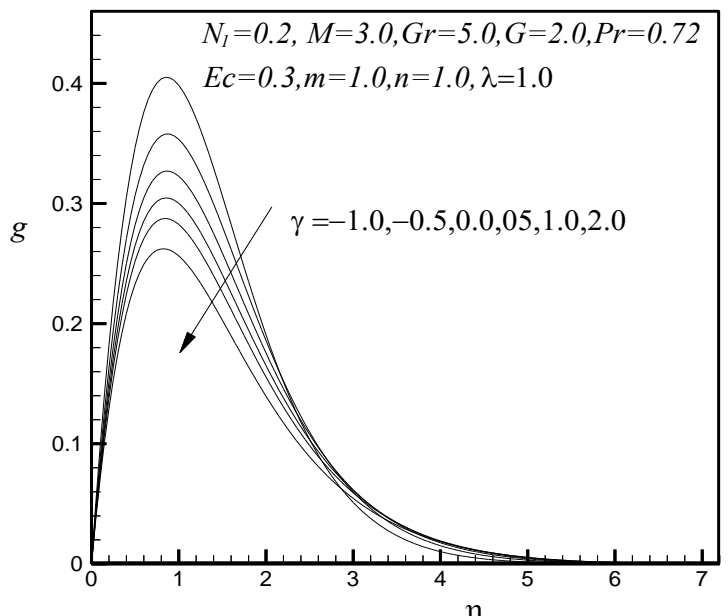

Fig. 35: Distribution of secondary velocity profiles for $\gamma$.

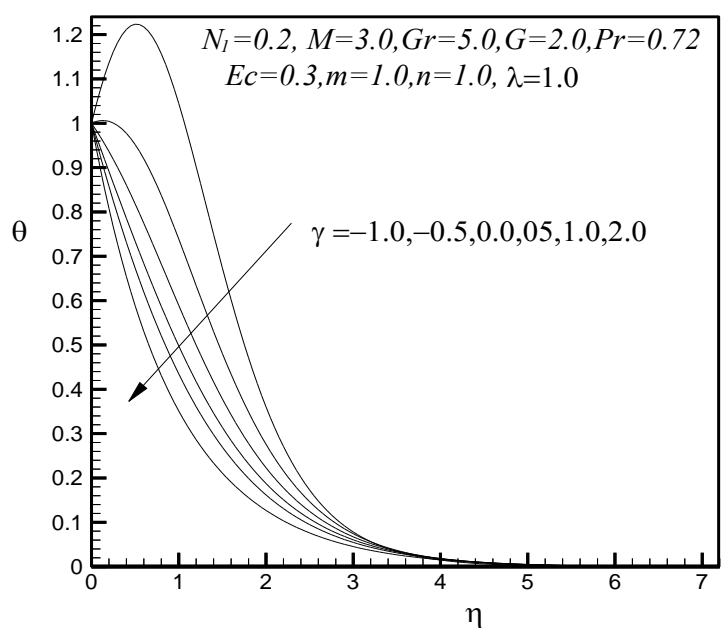

Fig. 37: Distribution of temperature profiles for $\gamma$. 


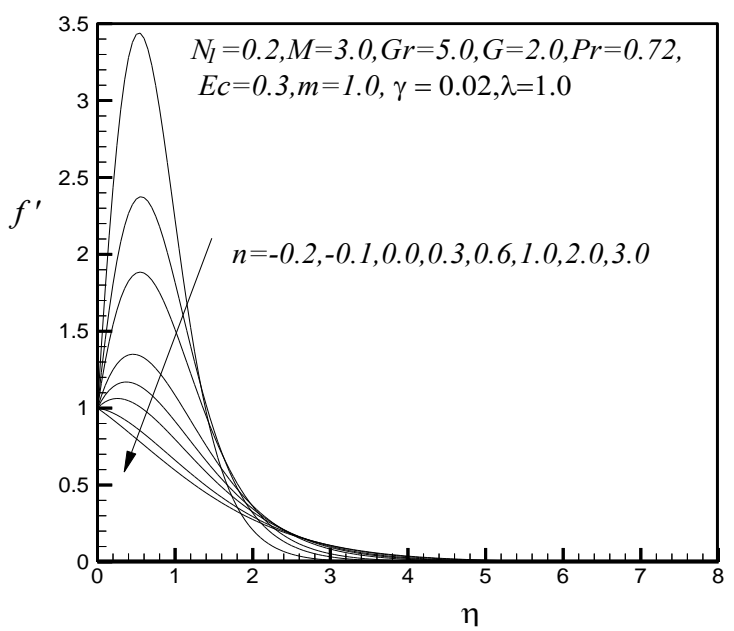

Fig. 38: Distribution of primary velocity profiles for $n$.

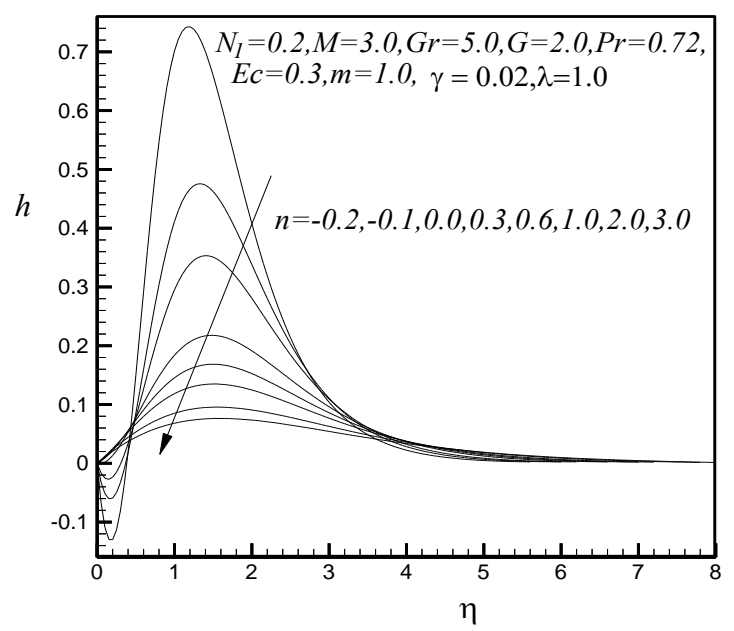

Fig. 40: Distribution of angular velocity profiles for $n$.

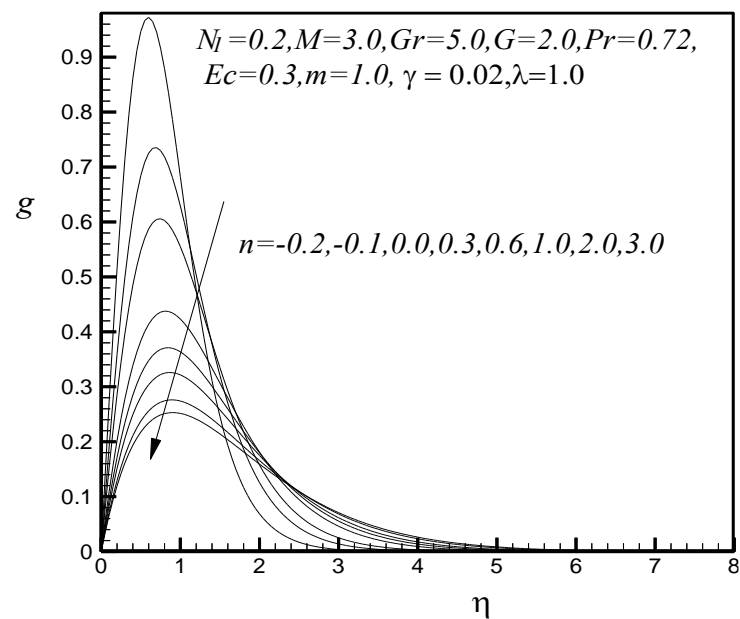

Fig. 39: Distribution of secondary velocity profiles for $n$.

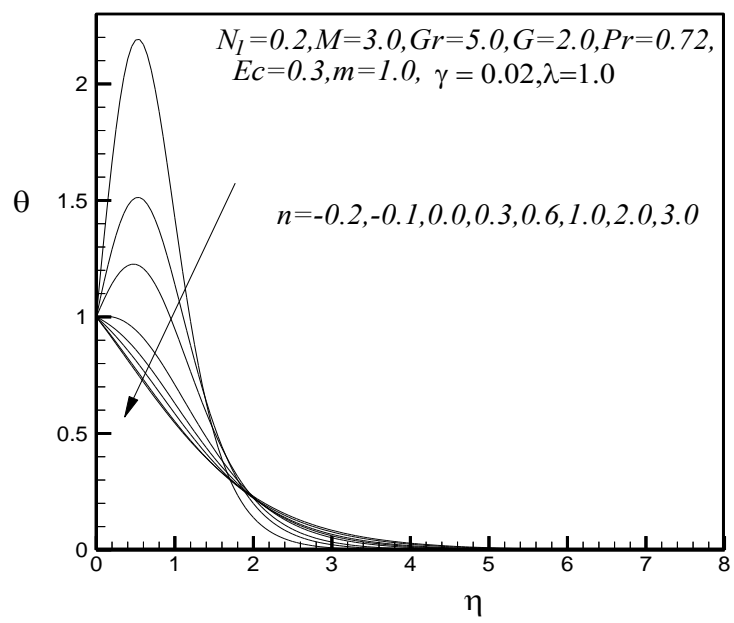

Fig. 41: Distribution of temperature profiles for $n$.

However maximum secondary velocity and angular velocity (micro-rotation) are attained further from the wall. The latter is related to the physical space available to micro-elements which is diminished near the wall and the magnitudes of secondary and angular velocity are respectively one and two orders lower than the primary velocity. Temperature is always a maximum at the wall itself (fig. 9).

Figs. 10-13 depict the influence of micropolar material parameter $\left(N_{1}\right)$ on the flow characteristics. This parameter features solely in the primary momentum equation (12), via the so-called "Eringen coupling term", $-N_{l} h^{\prime}$ which couples eqn. (12) with the micro-rotation equation (14). There is a separate coupling term in eqn. (14) connecting the primary velocity field to the angular velocity field, viz the $-f^{\prime \prime}$ term (a negative body force). Evidently this is a negative body force term and therefore an increase in $N_{l}$ will deplete the primary field further from the wall where greater gyratory motions can be sustained by micro-elements (close to the wall the reverse influence will be exerted). In consistency with this, fig. 10 demonstrates a strong reduction in primary velocity with greater $N_{l}$ values, a trend which is sustained into the free stream, far from the wall. The micropolar parameter, $N_{1}=K_{1} / v$ embodies the ratio of Eringen vortex viscosity to Newtonian kinematic viscosity. For cases where $N_{l}=0$, vortex 
viscosity vanishes $\left(K_{1} \rightarrow 0\right)$ and the micropolar gas reduces to a Newtonian gas (equation (14) also vanishes). For $N_{l}>0$ with progressively larger values of $N_{l}$, the concentration of microelements is elevated significantly, and rotary motions will be inhibited (fig. 12). The coupling parameter will have the same influence on the primary $\left(f^{\prime}\right)$ and angular velocity $(h)$ fields they are both boosted near the wall whereas further from the wall, after a critical distance, they are depressed. The influence of micropolar rheology on the gas flow is therefore nontrivial and the computations demonstrate that spin of suspended particles (micro-elements) should not be neglected in realistic (albeit laminar) gas dynamics simulations for MHD power generators. The depletion in primary momentum with greater $N_{1}$ values (stronger micropolarity of the gas) is also sustained by the secondary (cross) flow, as observed in fig. 11 , where significant deceleration is computed in particular at some distance from the wall in $g$ values. However, neither primary nor secondary flow reversal is generated as testified to by the consistently positive values of both velocity components in Figs. 10 and 11. A very weak decrease in temperature $(\theta)$ accompanies a significant increase in micropolar material parameter $\left(N_{1}\right)$, as depicted in Fig. 13. The thermal boundary layer thickness is therefore weakly reduced with greater micropolarity of the gas. Conversely primary and secondary momentum boundary layer thicknesses, and indeed micro-rotation boundary layer thickness will all be increased with greater values of $N_{1}$. The absence of $N_{1}$ in the energy (thermal) boundary layer equation (15) results in a very weak sensitivity of the thermal field to micropolar parameter; any influence is felt via the direct effect of micropolar vortex viscosity on the primary velocity and this in turn affects the secondary velocity. Both velocity fields are coupled to the energy equation (15) and the result is a minor one on temperatures.

Figs 14-17 present the evolution of primary, secondary and angular velocity component and temperature with buoyancy parameter $(G r) . G r$ is taken to be positive to represent cooling of the plate by free convection currents. It arises in the buoyancy term, $G r \theta$, in the primary momentum equation and thereby couples the primary velocity field with the temperature field (eqn. 15). Although linear in nature, it has a profound influence on the thermo-fluid dynamics processes. Primary velocity is evidently (fig. 14) boosted substantially near the wall (a velocity overshoot is again observed) and for some distance into the boundary layer transverse to the wall. At a critical distance from the wall, however, the influence of increasing $\mathrm{Gr}$ becomes inhibitive - it decelerates the primary flow, and this is maintained into the free stream. The influence of free convection currents decays further from the wall and this leads to retardation in the flow. $G r$ embodies the relative influence of the thermal buoyancy force to the viscous hydrodynamic force. For $G r>1$ the thermal buoyancy effect dominates viscous effects. A similar response for secondary velocity $(g)$ is computed in fig. 15. The crossflow velocity is significantly accelerated in close proximity to the wall and additionally thereafter; although the converse effect arises as with primary velocity, the critical point where this occurs is somewhat displaced closed to the wall than for the primary flow. Flow deceleration, with greater Grashof number, is therefore experienced for a greater proportion of the region transverse to the wall for the secondary velocity than for the primary velocity. Fig. 16 reveals that although initially there is a weak retardation in the angular velocity (micro-rotation) component, $h$, very quickly the effect of increasing Grashof number becomes assistive to the spin of micro-elements - $h$ values are markedly elevated throughout the majority of the boundary layer regime and attain asymptotically a non-zero value in the free stream. Peak micro-rotation however consistently arises quite soon in the boundary layer generally before transverse coordinate $(\eta)$ attains a value of 2 . The temperature function $(\theta)$ is strongly enhanced near the wall with greater Gr values. Thermal boundary layer thickness is therefore increased. However, for $\eta>1$, the contrary behavior is exhibited in fig. 17 and temperatures are found to be suppressed with greater Grashof numbers. 
Figs. 18-21 depict the variation in micropolar gas flow variables with Hall current parameter $(m)$. A weak acceleration in primary velocity (fig. 18) accompanies a large elevation in $m$, primarily in the vicinity of the wall. Further towards the free stream this trend is reversed, and deceleration is caused in the primary flow. This behavior is very different to that encountered in fully developed channel flows (see [54] for example) where consistent retardation in primary flow is induced with Hall current. Secondary (cross flow) velocity is affected enormously by the Hall current parameter $m$ as observed in Fig. 19. As elaborated earlier the supplementary body force arising in the primary momentum equation (12) due to the Hall effect, viz $-\frac{2}{n+1}\left[\frac{M \lambda}{1+m^{2} \lambda^{2}}\left(f^{\prime}+m \lambda g\right)\right]$, for $M>0$ will be negative. This will be impeded primary flow. However, in the secondary momentum (cross flow) conservation equation (13) the Hall body force term, viz $\frac{2}{n+1}\left[\frac{M \lambda}{1+m^{2} \lambda^{2}}\left(m \lambda f^{\prime}-g\right)\right]$ is effectively positive for $M>0$. This serves to aid the cross flow and manifests in substantial cross flow acceleration. It is further noteworthy that despite the $\mathrm{m}^{2}$ term appearing in the denominator in both body forces, the dominant effect is encapsulated in the numerator component terms, $m \lambda f^{\prime}$, which are directly proportional to $m$. The secondary velocity peak values are also fond to migrate further from the wall (plate) with increasing Hall parameter. In both figs 18, 19 backflows never arise, irrespective of the magnitude of Hall parameter. This implies that generator efficiency can be sustained even under high Hall effect scenarios, albeit in the secondary flow. Micro-rotation $(h)$ is also revealed in Fig. 20 to be assisted with greater Hall effect- angular velocity magnitudes are strongly enhanced and maximized much closer to the wall than to the free stream. A very minor reduction in micro-rotation arises as we approach the free stream. Overall however Hall current encourages the rotary motions of microelements. Temperature (Fig. 21) is very strongly suppressed with greater Hall current $(\mathrm{m})$ consistently throughout the boundary layer region. The appropriate term in eqn. (15) is the Ohmic dissipation term, viz $\frac{2}{n+1}\left[\frac{M \lambda}{1+m^{2} \lambda^{2}} \cdot E c\left(f^{\prime 2}+g^{2}\right)\right]$. Although the term is positive, the inverse square relationship with Hall parameter results in a significant depression in temperatures. Thermal boundary layer thickness is therefore also reduced. Again, the smooth decay of profiles in all the plots demonstrates that correct infinity boundary conditions have been imposed in the numerical computations.

Figs. 22-25 present the distributions for primary and secondary velocity, angular velocity and temperature distributions, respectively, with variation in the micro-rotational parameter $G=\frac{G_{1} B x^{n-1}}{K v}$. This parameter is also known as the Eringen spin gradient viscosity parameter. In the formulation employed for the angular momentum balance i.e. eqn. 14, the bulk spin torque term is neglected since an order of magnitude analysis [55] confirms that bulk spin viscosity is swamped by spin gradient viscosity effects. This parameter is always associated with the angular i.e. micro-rotation acceleration term $\left(h^{/ /}\right)$in the angular momentum balance, viz, $G((n+1) / 2) h^{\prime \prime}$. In the limit of $G \rightarrow 0$ couple stresses vanish, and the angular momentum equation is eliminated i.e. micro-structural effects vanish. Evidently the nature of $G$ is that it dominates the micro-rotation distributions, and this is confirmed in fig. 24 where with elevation in $G$ a dramatic deceleration is induced in angular velocity. In short, the microelement gyratory motions are inhibited with greater spin gradient viscosity. The influence of $G$ on primary and secondary velocity and also temperature profiles is trivial (figs. 22, 23, 25). These observations have also been reported in different micropolar transport problems by Gorla et al. [56], Hayat et al. [57] and more recently by Prasad et al. [58]. 
Figs. 26-29 present the evolution of micropolar gas flow characteristics with Prandtl number $(P r) . P r$ describes the relative influence of momentum and thermal diffusivities. Low $\mathrm{Pr}$ gases correspond to low density gases; higher $\mathrm{Pr}$ values are associated with denser micropolar gases and fluids. Since the present model is focused on gas flow, $P r=0.72$ has been prescribed in all the graphs, unless otherwise indicated. Here we address the additional cases of micropolar gases which may also be utilized in MHD power generators. $\mathrm{Pr}$ also defines the relative effectiveness of momentum diffusion in the velocity boundary layer to heat diffusion in the thermal boundary layer. These phenomena are of the same order of magnitude for the special case when $\operatorname{Pr}=1$. For $\operatorname{Pr}<1$ (e.g. 0.72) heat will diffuse at a significantly faster rate than the momentum. For $\operatorname{Pr}>1$, momentum will diffuse faster than the heat through the regime. The working fluid is a micropolar gas. Micropolar theory is not restricted to liquids. It is a generic theory which also applies to gases. Therefore, the Prandtl number $\operatorname{Pr}=0.7$ is a good approximation for the micropolar gas. Any fluid possessing viscosity (which included gases) can be modelled with micropolar mechanics. The micropolar theory is formulated significantly differently from conventional non-Newtonian models e.g. viscoelastic which focus on shear-stress relations and are applicable generally to polymeric or physiological liquids only and not gases. With greater $\operatorname{Pr}$ values figs 26, 27 and 29 reveal that primary and secondary velocity are both decreased markedly, and temperature is also strongly reduced. Primary and secondary boundary layer thicknesses are therefore increased whereas thermal boundary layer thickness is decreased. Conversely the microrotation (fig. 28) is found to be increased initially with greater Prandtl number near the wall; however further from the wall the angular velocity magnitudes are noticeably depressed with greater Prandtl number. Generally cooling is therefore achieved with higher Prandtl numbers and both primary and secondary flow is controlled better. With higher Prandtl number there is a much sharper descent in temperature profiles from the wall whereas the descent is much more gradual at low Prandtl number.

Figs. 30-33 illustrate the response in primary and secondary velocity, angular velocity and temperature functions to Eckert number $(E c) . E c$ expresses the relationship between the kinetic energy in the flow and the enthalpy difference. It therefore represents the conversion of kinetic energy into internal energy by work done against the viscous fluid stresses. Although this parameter is often used in high speed compressible flow, for example in rocket aerodynamics at very altitude, it has significance in high temperature incompressible flows, which are encountered in energy systems etc. Positive Eckert number implies cooling of the wall and therefore a transfer of heat to the fluid. Convection is enhanced. We observe that both primary and secondary flows are accelerated (Figs. 30, 31). Angular velocity (Fig. 32) is also enhanced significantly with greater Eckert number demonstrating that with greater viscous heating the micro-element rotary motions are intensified. The proportion of kinetic energy dissipated as heat manifests also with a strong elevation in temperature (Fig. 33). Thermal boundary layer thickness is therefore considerably increased with higher Eckert number.

Figs. 34-37 illustrate the response in primary and secondary velocity, angular velocity and temperature functions to non-isothermal power-law index parameter $(\gamma)$. This parameter relates to the variation in wall temperature and features in a single term in the energy equation (thermal boundary layer equation) i.e. eqn. (15), namely $-\frac{2}{n+1}\left[\gamma f^{\prime} \theta\right]$. For $\gamma>0$ the wall temperature increases with distance from the leading edge. For $\gamma<0$, wall temperature is reduced with distance from the leading edge. The case $\gamma=0$ corresponds to an isothermal wall. In the primitive equation (10) $\gamma$ arises in the wall thermal boundary condition as $T=T_{\mathrm{w}}=T_{\infty}+A x^{\gamma}$; however, following normalization of the equations it is featured only in 
the thermal transport equation (15). Primary and secondary velocities are both observed to be enhanced significantly with negative $\gamma$ values and conversely both velocity components are depressed with positive $\gamma$ values (figs. 34,35 ). Therefore, wall cooling is assistive to primary and secondary flow whereas wall heating is inhibitive. The contrary response is computed for micro-rotation (fig. 36) at least initially and close to the wall; further from the wall however negative power-law index is again observed to accelerate the flow whereas positive powerlaw index induces retardation in angular velocity. Temperatures are observed to be consistently boosted with greater negative $\gamma$ values whereas they are strongly suppressed with positive $\gamma$ values. A significant temperature overshoot is also observed for $\gamma=-1.0$, close to the wall.

Figs. 38-41 finally display the micropolar gas flow characteristics with various wall distention parameters $(n)$. This parameter arises throughout all the boundary layer equations (12)-(15). Generally, with greater negative $n$ values, corresponding to deceleration of the wall (contraction), primary and secondary velocity components, micro-rotation and temperature are all substantially increased. With greater positive $n$ values (decelerating stretching wall), the contrary behavior is generally computed. All the flow characteristics peak near the plate; however, after some distance from the wall the influence of negative/positive $n$ values is reversed. Once again asymptotically smooth decays to the free stream are achieved for all distributions.

\section{Conclusions}

In the present paper, the effects of Hall current and viscous and Joule (Ohmic) electromagnetic dissipation on the non-isothermal micropolar boundary layer flow and heat transfer from a nonlinear stretching/contracting surface permeated by a strong magnetic field have been studied. The model is representative of near-wall Hall MHD power generator flows utilizing electrically conducting micropolar gaseous working fluids. The governing momentum and energy equations have been transformed to a system of non-linear ordinary differential equations by employing the appropriate coordinate transformations. The resulting nonlinear, multi-degree, boundary value problem has been solved numerically with an efficient Runge-Kutta shooting algorithm. Validation of solutions has been achieved with both the Homotopy analysis method (HAM) and Adomian decomposition method (ADM), both semi-numerical approaches achieving excellent correlation. A parametric study of the influence of the emerging thermophysical and magnetohydrodynamic parameters, namely Hartmann number $(M)$, Eringen vortex viscosity parameter $\left(N_{1}\right)$, Grashof number $(G r)$, Hall parameter $(m)$, microrotational parameter $(G)$, Prandtl number $(\operatorname{Pr})$, Eckert number $(E c)$, nonisothermal power-law exponent $(\gamma)$ and wall stretching/contraction parameter $(n)$ on the primary, secondary velocity (momentum), angular velocity (microrotation) and temperature profiles has been conducted. The present computations have revealed that the Hartmann magnetic body force parameter very effectively controls the heat transfer characteristics, Grashof number (buoyancy parameter) has a significant effect on all velocity fields and temperature and Hall current generally accelerates the secondary (cross) flow. Furthermore, it is found that increasing micro-rotational (Eringen spin gradient viscosity) parameter primarily suppresses angular velocity, increasing Eckert number strongly elevates all velocity functions (primary, secondary and angular) and heats the boundary layer, and stretching/contracting parameter (non-linear wall geometric) parameter substantially modifies all thermofluid characteristics. The present simulation has been confined to steady-state flow. Future investigations will consider transient [59] effects which are also of relevance to MHD Hall generators and will be reported imminently. 


\section{Acknowledgements}

This research did not receive any specific grant from funding agencies in the public, commercial, or not- for profit sectors. The authors are grateful to the reviewers for their comments which have clarified aspects of the work and improved the manuscript.

\section{References}

[1] Macheret, S., Shneider, M., Candler, G., Moses, R., Kline, J.: Magnetohydrodynamic power generation for planetary entry vehicles. AIAA-2004-2560 35th AIAA Plasmadynamics and Lasers Conference, Portland, Oregon, June 28-1 (2004).

[2] Peng, Y., Lin, Z., Zhao, L., Sha, C., Li, R., Xu, Y., Li, J.: Analysis of liquid metal MHD wave energy direct conversion system. Proc. $18^{\text {th }}$ Int. Offshore Polar Eng Conf, Vancouver, Canada, ISOPE. 1, 388-392 (2008).

[3] Hu, L.L., Kobayashi, H., Okuno, Y.: Analyses on response of a liquid metal MHD power generation system to various external inputs. IEEE Trans. Elec Electron Eng. 10, 268-273 (2015).

[4] Hardianto, T., Harada, N.: Three-dimensional flow analysis in a Faraday-type MHD generator. IEEE Trans. Ind. Appl. 44, 90-100 (2008).

[5] Rajesh, V., Mallesh, M.P., Anwar Bég, O.: Transient MHD free convection flow and heat transfer of nanofluid past an impulsively started vertical porous plate in the presence of viscous dissipation. Proc. Mat. Sci. 10, 80-89 (2015).

[6] Murakami, T., Okuno, Y.: High density energy conversion using compact magnetohydrodynamic electrical power generators. Appl. Phys. Letters. 91, 161506 (2007).

[7] Norouzi, M., Davoodi, M., Anwar Bég, O., Joneidi, A.A.: Analysis of the effect of normal stress differences on heat transfer in creeping viscoelastic Dean flow. Int. J. Ther. Sci. 69, 61-69 (2013)

[8] Tripathi, D., Anwar Bég, O.: A numerical study of oscillating peristaltic flow of generalized Maxwell viscoelastic fluids through a porous medium. Transport in Porous Media. 95, 337-348 (2012).

[9] Prasad, V.R., Gaffar, S.A., Reddy, E.K., Anwar Bég, O.: Flow and heat transfer of Jefferys non-Newtonian fluid from a horizontal circular cylinder. AIAA J. Thermophysics and Heat Transfer. 28(4), 764-770 (2014).

[10] Eringen, A.C.: Simple microfluids. Int. J. Eng. Sci. 2, 205-217 (1964).

[11] Eringen, A.C.: Theory of micropolar fluids. J. Math. Mech. 16, 1-18 (1966).

[12] Migun, N.P.: Experimental method of determining parameters characterizing the microstructure of micropolar liquids. J. Eng. Phys. 41, 832-835 (1981).

[13] Anwar Bég, O., Bhargava, R., Rashidi, M.M.: Numerical Simulation in Micropolar Fluid Dynamics. Germany: Lambert Academic Press. (288 pages) (2011).

[14] Guram, G.S., Smith, A.C.: Stagnation flows of micropolar fluids with strong and weak interactions. Comput. Math. App. 6, 213-233 (1980). 
[15] Gorla, R.S.R., Takhar, H.S.: Boundary layer flow of micropolar fluid on rotating axisymmetric surfaces with a concentrated heat source. Acta Mechanica. 105, 1-10 (1994).

[16] Char, M.I., Chang, C.L.: Effect of wall conduction on natural convection flow of micropolar fluids along a flat plate. Int. J. Heat Mass Transfer. 40, 3641-3652 (1997).

[17] Takhar, H.S., Bhargava, R., Agrawal, R.S., Balaji, A.V.S.: Finite element solution of a micropolar fluid flow and heat transfer between two porous disks. Int. J. Eng. Sci. 38, 1907-1922 (2000).

[18] El-Arabawy, H.A.M.: Effect of suction/injection on the flow of a micropolar fluid past a continuously moving plate in the presence of radiation. Int. J. Heat and Mass Transfer. 46, 1471-1477 (2003).

[19] Hassanien, I.A., Essawy, A.H., Moursy, N.M.: Natural convection flow of micropolar fluid from a permeable uniform heat flux surface in porous medium. App. Math. Comp. 152, 323-335 (2004).

[20] Anwar Bég, O., Prasad, V.R., Vasu, B., Reddy, N.B., Li, Q., Bhargava, R.: Free convection heat and mass transfer from an isothermal sphere to a micropolar regime with Soret/Dufour effects. Int. J. Heat and Mass Transfer. 54, 9-18 (2011).

[21] Damesh, R.A., Al-Azab, T.A., Shannak, B.A., Al Husein, M.: Unsteady natural convection heat transfer of micropolar fluid over a vertical surface with constant heat flux. Turkish J. Eng. Env. Sci. 31, 225-233 (2007).

[22] Anwar Bég, O., Rashidi, M.M., Bég, T.A., Asadi, M.: Homotopy analysis of transient magneto-bio-fluid dynamics of micropolar squeeze film: a model for magneto biorheological lubrication. J. Mech. Med. Biol. 2(3), 1250051.1-1250051.21 (2012).

[23] Zueco, J., Anwar Bég, O., Takhar, H.S.: Network numerical analysis of magnetomicropolar convection through a vertical circular non-Darcian porous medium conduit. Comput. Mat. Sci. 46(4), 1028-1037 (2009).

[24] Rahman, M.M., Uddin, M.J., Aziz, A.: Convective flow of micropolar fluid in a porous medium with variable electric conductivity, surface heat flux and non-uniform heat source (or sink). Int. J. Energy Tech. 25, 1-18 (2010).

[25] Sherief, H.H., Faltas, M.S., Ashmawy, E.A.: Exact solution for the unsteady flow of a semi-infinite micropolar fluid. Acta Mech. Sin. 27, 354-359 (2011).

[26] Anwar Bég, O.: Numerical methods for multi-physical magnetohydrodynamics, Ch.1, pp. 1-110, New Developments in Hydrodynamics Research, (Eds: Maximiano J. Ibragimov and Miroslava A. Anisimov), Nova Science, New York (2012).

[27] Elie, S., Ghaddar, N., Chaaban, F.: Evaluation of the Hall parameter of electrolyte solutions in thermosyphonic MHD flow. Int. J. Eng. Sci. 40, 2041-2056 (2002).

[28] Anwar Bég, O., Ghosh, S.K., Bég, Tasveer A., Applied Magnetofluid Dynamics: Modelling and Computation. Lambert Academic Press, Germany (2011).

[29] Aurangzeeb., Shafie, S.: Effects of Soret and Dufour on unsteady MHD flow by mixed convection over a vertical surface in porous media with internal heat generation, chemical reaction and Hall current. Canadian J. Sci. Eng. Math. 2, 153-162 (2011).

[30] Ferdows, M., Jahan, E., Hamad, M.M., Masahiro, O.T.A.: Effects of Hall and ion-slip currents on free convective heat transfer flow past a vertical plate considering slip conditions. Canadian J. Sci. Eng. Math. 2, 70-76 (2011). 
[31] Anwar Bég, O., Sim, L., Zueco, J., Bhargava, R.: Numerical study of magnetohydrodynamic viscous plasma flow in rotating porous media with Hall currents and inclined magnetic field influence. Commun. Nonlinear Sci. Numer. Simult. 15, 345-359 (2010).

[32] Kumar, R., Chand, K.: Effect of slip conditions and hall current on unsteady MHD flow of a viscoelastic fluid past an infinite vertical porous plate through porous medium. Int. J. Eng. Sci. Tech. 3, 3124-3133 (2011).

[33] Ghosh, S.K., Anwar Bég, O., Zueco, J., Prasad, V.R.: Transient hydromagnetic flow in a rotating channel permeated by an inclined magnetic field with magnetic induction and Maxwell displacement current effects. ZAMP: J. Appl. Math. Phys. 61, 147-169 (2010).

[34] Raju, M.C., Reddy, N.A., Varma, S.V.K.: Hall-current effects on unsteady magnetohydrodynamics flow between stretching sheet and an oscillating porous upper parallel plate with constant suction. Therm. Sci. 15, 527-536 (2011).

[35] Anwar Bég, O., Gaffar, S.A., Prasad, V.R., Uddin, M.J.: Computational solutions for nonisothermal, nonlinear magneto-convection in porous media with Hall/ion slip currents and Ohmic dissipation. Eng. Sci. Tech-An Int. J. 19(1), 377-394 (2015).

[36] Ghara, N., Maji, S.L., Das, S., Jana, R., Ghosh, S.K.: Effects of Hall current and ion-slip on unsteady MHD Couette flow. Open J. Fluid Dynamics. 2, 1-13 (2012).

[37] Hayat, T., Hendi, F.A.: Thermal-diffusion and diffusion-thermo effects on MHD threedimensional axisymmetric flow with Hall and ion-slip currents. J. American Sci. 8, 284294 (2012).

[38] Motsa, S.S., Shateyi, S.: The effects of chemical reaction, Hall, and ion-slip currents on MHD micropolar fluid flow with thermal diffusivity using a novel numerical technique. J. Appl. Math. 2012, 1-30 (2012).

[39] Sparrow EM, and Yu, HS., Local non- similarity thermal boundary- layer solutions. ASME Journal of Heat Transfer, 93, 328-334 (1971)

[40] Nachtsheim, P.R., Swigert, P.: Satisfaction of the asymptotic boundary conditions in numerical solution of the systems of non-linear equations of boundary layer type. NASA TN D-3004. Washington. D.C. USA (1965).

[41] Anwar Bég, O., Uddin, M.J., Rashidi, M.M., Kalyani, N.: Double-diffusive radiative magnetic mixed convective slip flow with Biot and Richardson number effects. J. Eng. Thermophys. 23(2), 79-97 (2014).

[42] Anwar Bég, O., Ferdows, M., Islam, S., Islam, M.N.: Numerical simulation of Marangoni magnetohydrodynamic bio-nanofluid convection from a non-isothermal surface with magnetic induction effects: a bio-nanomaterial manufacturing transport model. J. Mech. Med. Biol. 14, 1450039.1-1450039.32 (2014).

[43] Uddin, M.J., Anwar Bég, O., Amin, N.S.: Hydromagnetic transport phenomena from a stretching or shrinking nonlinear nanomaterial sheet with Navier slip and convective heating: a model for bio-nano-materials processing. J. Magn. Magn. Mat. 368, 252-261 (2014).

[44] Ferdows, M., Khan, M.S., Anwar Bég, O., Azad, M.A.K., Alam, M.M.: Numerical study of transient magnetohydrodynamic radiative free convection nanofluid flow from a stretching permeable surface. Proc. IMechE-Part E: J. Process Mech. Eng. 228(3), 181196 (2014). 
[45] Liao, S.J.: Beyond Perturbation: Introduction to the Homotopy Analysis Method. CRC Press, USA (2003).

[46] Anwar Bég, O., Mabood, F., Islam, M.N.: Homotopy simulation of nonlinear unsteady rotating nanofluid flow from a spinning body. Int. J. Eng. Math. 2015, Article ID:272079 (2015).

[47] Chaht, F.L., Kaci, A., Houari, M.S.A., Tounsi, A., Anwar Bég, O., Mahmoud, S.R.: Bending and buckling analyses of functionally graded material (FGM) size-dependent nanoscale beams including the thickness stretching effect. Steel and Composite Structures. 18(2), 425-442 (2015).

[48] Mehmood, A., Asif, A., Takhar, H.S., Anwar Bég, O., Islam, M.N., Wilson, L.S.: Unsteady Von Kármán swirling flow: analytical study using the Homotopy Method. Int. J. Appl. Math. Mech. 6(2), 67-84 (2010).

[49] Anwar Bég, O., Tripathi, D., Sochi, T., Gupta, P.K.: Adomian decomposition method (ADM) simulation of magneto-bio tribological squeeze film with magnetic induction effects. J. Mech. Med. Biol. 15, 1550072.1-1550072.23 (2015).

[50] Gupta, D., Kumar, L., Anwar Bég, O., Singh, B.: Finite element simulation of nonlinear magneto-micropolar stagnation point flow from a porous stretching sheet with prescribed skin friction. Comput. Therm. Sci. 7(1), 1-14 (2014).

[51] Adomian, G.: Solving Frontier Problems in Physics, USA: Springer (1994).

[52] Shamshuddin, MD., Anwar Bég, O., Sunder Ram, M., Kadir, A.: Finite element computation of multi-physical micropolar transport phenomena from an inclined moving plate in porous media, Ind. J. Phys. 92(2), 215-230 (2017).

[53] Gorla RSR, Sidawi I. Free convection on a vertical stretching surface with suction and blowing. Appl Sci Res. 52(3):247-257 (1994).

[54] Anwar Bég, O., Zueco, J., Takhar, H.S.: Unsteady magnetohydrodynamic HartmannCouette flow and heat transfer in a Darcian channel with Hall current, ion slip, viscous and Joule heating effects: Network numerical solutions. Commun. Nonlinear Sci. Numer. Simulat. 14, 1082-1097 (2009).

[55] Hoffmann, K.H., Marx, D., Botkin, N.D.: Drag on spheres in micropolar fluids with nonzero boundary conditions for microrotations. J. Fluid Mech. 590, 319-330 (2007).

[56] Gorla, R.S.R., Slaouti, A., Takhar, H.S.: Free convection in micropolar fluids over a uniformly heated vertical plate, Int. J. Numerical Methods for Heat Fluid Flow. 8, 504518 (1998).

[57] Hayat, T., Nawaz, M., Obaidat, S., Axisymmetric magnetohydrodynamic flow of micropolar fluid between unsteady stretching surfaces. App. Math. Mech. 32, 361-374 (2011).

[58] Prasad, V.R., Gaffar, S.A., Anwar Bég, O.: Heat and mass transfer of a nanofluid from a horizontal cylinder to a micropolar fluid. AIAA J. Thermophysics Heat Transfer. 29(1), 127-139 (2015).

[59] Anwar Bég, O., Rawat, S., Zueco, J., Osmond, L., Gorla, R.S.R.: Finite element and network electrical stimulation of rotating magnetofluid flow in nonlinear porous media with inclined magnetic field and Hall currents. Theor. Appl. Mech. 41(1), 1-35 (2014). 